\title{
A Revised Broad-Line Region Radius and Black Hole Mass for the Narrow-Line Seyfert 1 NGC 4051
}

\author{
K. D. Denney ${ }^{1}$, L. C. Watson ${ }^{1}$, B. M. Peterson ${ }^{1,2}$, R. W. Pogge ${ }^{1,2}$, D. W. Atlee ${ }^{1}$, \\ M. C. Bentz ${ }^{1,3}$, J. C. Bird ${ }^{1}$, D. J. Brokofsky ${ }^{4,5}$, M. L. Comins ${ }^{1,6}$, M. Dietrich ${ }^{1}$, \\ V. T. Doroshenko ${ }^{7,8,16}$, J. D. Eastman ${ }^{1}$, Y. S. Efimov ${ }^{8}$, C. M. Gaskell ${ }^{4,9}$, C. H. Hedrick ${ }^{4,6}$, \\ S. A. Klimanov ${ }^{8,16}$, E. S. Klimek ${ }^{4,10}$, A. K. Kruse ${ }^{4}$, J. Lamb ${ }^{11}$, K. Leighly ${ }^{12}$, T. Minezaki ${ }^{13}$, \\ S. V. Nazarov ${ }^{8.16}$, E. A. Petersen ${ }^{4}$, P. Peterson ${ }^{14}$, S. Poindexter ${ }^{1}$, Y. Sakata ${ }^{15}$ \\ K. J. Schlesinger ${ }^{1}$, S. G. Sergeev ${ }^{7,16}$, J. J. Tobin ${ }^{11}$, C. Unterborn ${ }^{1}$, M. Vestergaard ${ }^{17,18}$, \\ A. E. Watkins ${ }^{4}$, and Y. Yoshii ${ }^{13,19}$
}




\begin{abstract}
We present the first results from a high sampling rate, multi-month reverberation mapping campaign undertaken primarily at MDM Observatory with
\end{abstract}

\footnotetext{
${ }^{1}$ Department of Astronomy, The Ohio State University, 140 West 18th Avenue, Columbus, OH 43210; denney, watson, peterson, pogge@astronomy.ohio-state.edu

${ }^{2}$ Center for Cosmology and AstroParticle Physics, The Ohio State University, 191 West Woodruff Avenue, Columbus, OH 43210

${ }^{3}$ Present address: Dept. of Physics and Astronomy, 4129 Frederick Reines Hall, University of California at Irvine, Irvine, CA 92697-4575; mbentz@uci.edu

${ }^{4}$ Department of Physics \& Astronomy, University of Nebraska, Lincoln, NE 68588-0111.

${ }^{5}$ Deceased, Sept. 13, 2008

${ }^{6}$ Present address: Astronomy and Astrophysics Department, Pennsylvania State University, 525 Davey
} Laboratory, University Park, PA 16802

${ }^{7}$ Crimean Laboratory of the Sternberg Astronomical Institute, p/o Nauchny, 98409 Crimea, Ukraine; vdorosh@sai.crimea.ua

${ }^{8}$ Crimean Astrophysical Observatory, p/o Nauchny, 98409 Crimea, Ukraine; sergeev, efim@crao.crimea.ua, sergdave2004@mail.ru,nazarastron2002@mail.ru

${ }^{9}$ Present address: Astronomy Department, University of Texas, Austin, TX 78712-0259; gaskell@astro.as.utexas.edu

${ }^{10}$ Present address: Astronomy Department, MSC 4500, New Mexico State University, PO BOX 30001, La Cruces, NM 88003-8001

${ }^{11}$ Department of Astronomy, University of Michigan, 500 Church St., Ann Arbor, MI 48109-1040

${ }^{12}$ Homer L. Dodge Department of Physics and Astronomy, The University of Oklahoma, 440 W. Brooks St., Norman, OK 73019

${ }^{13}$ Institute of Astronomy, School of Science, University of Tokyo, 2-21-1 Osawa, Mitaka, Tokyo 181-0015, Japan; minezaki, yoshii@ioa.s.u-tokyo.ac.jp

${ }^{14}$ Ohio University, Department of Physics and Astronomy, Athens, OH 45701-2979

${ }^{15}$ Department of Astronomy, School of Science, University of Tokyo, 7-3-1 Hongo, Bunkyo-ku, Tokyo 113-0013, Japan

${ }^{16}$ Isaak Newton Institute of Chile, Crimean Branch, Ukraine

${ }^{17}$ Steward Observatory, The University of Arizona, 933 North Cherry Avenue, Tucson, AZ 85721

${ }^{18}$ Present address: Department of Physics and Astronomy, Tufts University, Medford, MA 02155

${ }^{19}$ Research Center for the Early Universe, School of Science, University of Tokyo, 7-3-1 Hongo, Bunkyo-ku, Tokyo 113-0033, Japan 
supporting observations from telescopes around the world. The primary goal of this campaign was to obtain either new or improved $\mathrm{H} \beta$ reverberation lag measurements for several relatively low luminosity AGNs. We feature results for NGC 4051 here because, until now, this object has been a significant outlier from AGN scaling relationships, e.g., it was previously a $\sim 2-3 \sigma$ outlier on the relationship between the broad-line region (BLR) radius and the optical continuum luminosity - the $R_{\mathrm{BLR}}-L$ relationship. Our new measurements of the lag time between variations in the continuum and $\mathrm{H} \beta$ emission line made from spectroscopic monitoring of NGC 4051 lead to a measured BLR radius of $R_{\mathrm{BLR}}=1.87_{-0.50}^{+0.54}$ light days and black hole mass of $M_{\mathrm{BH}}=\left(1.73_{-0.52}^{+0.55}\right) \times 10^{6} M_{\odot}$. This radius is consistent with that expected from the $R_{\mathrm{BLR}}-L$ relationship, based on the present luminosity of NGC 4051 and the most current calibration of the relation by Bentz et al. (2009a). We also present a preliminary look at velocityresolved $\mathrm{H} \beta$ light curves and time delay measurements, although we are unable to reconstruct an unambiguous velocity-resolved reverberation signal.

Subject headings: galaxies:active — galaxies: nuclei — galaxies: Seyfert

\section{INTRODUCTION}

Recent theoretical and observational studies have provided strong evidence suggesting a connection between supermassive black hole (SMBH) growth and galaxy evolution (e.g., Bennert et al. 2008; Somerville et al. 2008; Shankar et al. 2009; Hopkins \& Hernquist 2009). To better understand this connection, we need more direct measurements of SMBH masses across cosmological distances. Unfortunately, measuring SMBH masses directly with dynamical methods requires high angular resolution, so use of these methods is limited to relatively nearby sources. This resolution problem can be obviated by studying SMBHs in type 1 active galactic nuclei (AGNs). In this case, the technique of reverberation mapping (Blandford \& McKee 1982; Peterson 1993) may be applied to measure the SMBH mass, as has been done for more than 3 dozen type 1 AGNs to date (e.g., see recent compilation by Peterson et al. 2004).

Reverberation mapping relies on time resolution rather than angular resolution, since it takes advantage of the presence of a time delay, $\tau$, between continuum and emission line flux variations observed through spectroscopic monitoring. This time delay corresponds to the light travel time across the broad-line region (BLR), and thus measurements of $\tau$ provide an estimate of the size of the region, $R_{\mathrm{BLR}}=c \tau$. Because the BLR gas is well within the sphere of influence of the black hole and studies have provided evidence for virialized motions 
within this region (e.g., Peterson et al. 2004, and references therein), $R_{\mathrm{BLR}}$ can be related to the mass of the SMBH through the velocity dispersion of the BLR gas.

Although stellar and gas dynamical modeling and reverberation mapping are invaluable for measuring SMBH masses directly, these methods are observationally taxing, due to resolution requirements for dynamical methods and time requirements for reverberation mapping campaigns. It is currently impossible to measure SMBH masses directly for large statistical samples of galaxies. However, these direct mass measurements have led to the discovery of scaling relationships that relate SMBH mass to other galaxy or AGN observables that can be used to investigate the connection between SMBH mass and galaxy evolution. In particular, some relations show connections between properties of the SMBH (i.e., its mass) and global properties of the host galaxy. Examples include the correlation between SMBH mass and bulge/spheroid stellar velocity dispersion, i.e. the $M_{\mathrm{BH}}-\sigma_{\star}$ relation for AGNs (Gebhardt et al. 2000b; Ferrarese et al. 2001; Onken et al. 2004; Nelson et al. 2004) and quiescent galaxies (Ferrarese \& Merritt 2000; Gebhardt et al. 2000a; Tremaine et al.|2002), and the correlation between SMBH mass and galaxy bulge luminosity (Kormendy \& Richstone 1995; Magorrian et al. 1998; Wandel 2002; Graham 2007; Bentz et al. 2009b). Other relations connect various AGN properties. One such relation is the correlation between black hole mass and optical luminosity (Kaspi et al. 2000; Peterson et al. 2004), which relates directly to the accretion rates of AGNs. There is also a correlation between BLR radius and AGN luminosity, i.e., the $R_{\mathrm{BLR}}-L$ relation (Kaspi et al. 2000, 2005; Bentz et al. 2006, 2009a), which has proven to be very powerful for making indirect SMBH mass estimations from single-epoch spectra (e.g., Vestergaard 2002, 2004; Corbett et al. 2003; Kollmeier et al. 2006; Vestergaard et al. 2008; Shen et al. 2008a, b; Fine et al. 2008). These indirect mass estimates can then be related to other properties of the host galaxy through direct measurements or separate scaling relations.

Although scaling relations have become widely used for statistical studies, it is important to understand that the indirect mass estimates determined by these relations are only as reliable as the direct mass measurements used to calibrate them. Therefore, establishing a secure calibration across a wide dynamic range in parameter space and better understanding any intrinsic scatter in these relations is essential. To accomplish this, we must continue to make new direct measurements as well as to check previous results that are, for one reason or another, suspect.

NGC 4051, an SAB(rs)bc galaxy with a narrow-line Seyfert 1 (NLS1) nucleus at redshift $z=0.00234$, is a case in point. Measurements of the BLR radius and optical luminosity (Peterson et al. 2000, 2004) place it above the $R_{\mathrm{BLR}}-L$ relation, i.e., the BLR radius is too large for its luminosity (cf. Figure 2 of Kaspi et al. 2005). It also appears to be accreting 
mass at a lower Eddington rate than other NLS1s (cf. Figure 16 of Peterson et al. 2004). These two anomalies together suggest that perhaps the BLR radius has been overestimated by Peterson et al. (2000, 2004); indeed an independent reverberation measurement of the BLR radius in NGC 4051 by Shemmer et al. (2003, hereafter S03) is about half the value measured by Peterson et al. (2000, hereafter P00). Furthermore, neither the P00 nor S03 data sets are particularly well sampled on short time scales, so neither set is suitable for detection of smaller time lags (e.g., $\lesssim 2-3$ days). In addition, Russell (2003) reports a TullyFisher distance to NGC 4051 that is $\sim 50 \%$ larger than that inferred from its redshift (i.e., 15.2 Mpc versus 10.0 Mpc, respectively). This suggests that the luminosity derived in past studies from the redshift could be an underestimate and might also be a contributing factor to the placement of NGC 4051 above the $R_{\mathrm{BLR}}-L$ relation.

In this work, we present an analysis of new, optical spectroscopic and photometric observations of NGC 4051, which represent the first results from a densely sampled reverberation mapping campaign that began in early 2007. The campaign spanned more than 4 months, during which time we consistently obtained multiple photometric observations per night and spectroscopic observations nearly every night from a combination of five different observatories around the globe. The immediate goal of this campaign is to remeasure the $\mathrm{H} \beta$ reverberation lag measurements for several objects on the low-luminosity end of $R_{\mathrm{BLR}}-L$ scaling relationship with poorly determined reverberation lags and, consequently, poorly determined black hole masses. We will also add to the overall sample of reverberation mapped AGNs by measuring lags for new objects. Another goal for this extensive collection of homogeneous data is to reconstruct the observed response of the $\mathrm{H} \beta$ emission line to an outburst from the variable continuum source by modeling the response as a function of both line-ofsight velocity and light travel time, i.e., a "velocity-delay map" (for a tutorial, see Peterson 2001; Horne et al. 2004). Creation of a velocity-delay map will provide novel insight into the structure and kinematics of the BLR. Though we have not yet attempted to reconstruct a full velocity-delay map, we present preliminary velocity-resolved lag measurements for NGC 4051. Complete results for NGC 4051 and other campaign targets will be presented in future work.

\section{Observations and Data Analysis}

Most data acquisition and analysis practices employed here follow closely those described

by Denney et al. (2006) and laid out by Peterson et al. (2004). The reader is referred to these works for additional details and discussions. Throughout this work, we assume the following cosmological parameters: $\Omega_{m}=0.3, \Omega_{\Lambda}=0.70$, and $H_{0}=70 \mathrm{~km} \mathrm{sec}^{-1} \mathrm{Mpc}^{-1}$. 


\subsection{Spectroscopy}

Spectra of the nuclear region of NGC 4051 were obtained from both the 1.3-meter telescope at MDM Observatory and the 2.6-meter Shajn telescope of the Crimean Astrophysical Observatory (CrAO). The MDM observations utilized the Boller and Chivens CCD spectrograph, where 86 observations were taken over the course of 89 nights between JD2454184 and JD2454269, targeting the $\mathrm{H} \beta \lambda 4861$ and [O III] $\lambda \lambda 4959,5007$ emission line region of the optical spectrum. The position angle was set to $0^{\circ}$, with a slit width of $5^{\prime \prime} .0$ projected on the sky, resulting in a spectral resolution of $7.6 \AA$ across this emission-line region. Figure 1 shows the mean and rms spectra of NGC 4051 based on the MDM observations. We acquired 22 CrAO spectra over 34 nights between JD2454266 and JD2454300 with the Nasmith spectrograph and SPEC-10 $1340 \times 100$ pixel CCD. For these observations a 3'.0 slit was utilized, with a $90^{\circ}$ position angle. Spectral wavelength coverage for this data set was from $\sim 3800-6000 \AA$, with a dispersion of $1.8 \AA$ pix and a spectral resolution of $7.5 \AA$. Note that (1) the dispersion varies with wavelength: the value $1.8 \AA /$ pix is given for $5100 \AA$, and (2) the real wavelength coverage is slightly greater than given but the red and blue edges of the CCD frame are unusable (too low $\mathrm{S} / \mathrm{N}$ ratio) because of vignetting.

A relative flux calibration of each set of spectra was performed based on the constancy of the narrow [O III] $\lambda 5007$ line flux. Because this line emission originates in the extended, lowdensity narrow line region, it can be assumed constant over the timescale of this campaign and therefore serves as the basis for a reliable relative flux calibration. However, the data quality is not identical from night to night due to, e.g., seeing, weather conditions, atmospheric transparency, etc. This affects not only the integrated line flux in each observation, but also properties of the spectrum such as $\mathrm{S} / \mathrm{N}$ and resolution. Consequently, we employ the spectral scaling algorithm of van Groningen \& Wanders (1992) for the [O III] $\lambda 5007$ flux calibration. This algorithm determines the best scaling through $\chi^{2}$ minimization of residuals rather than simply calculating a simple multiplicative scale factor to scale the spectral fluxes. Following this method, we created a reference spectrum by averaging all spectra from a given data set. We then formed a difference spectrum by subtracting the reference spectrum from each individual spectrum. The algorithm uses a least squares method to minimize the residuals of the [O III $]$ 5007 line flux in each difference spectrum by making small zero-point wavelength calibration adjustments, correcting for resolution differences, and applying a multiplicative scale factor to the [O III] $\lambda 5007$ line flux of the individual spectrum. Because this method is based on minimizing residuals between each individual spectrum and the reference spectrum, there is a small residual dispersion in the line fluxes after calibration. This dispersion is related to the data quality and the ability of the scaling algorithm to mitigate night to night differences between individual spectra, related largely to seeing and S/N. Tests of the original

algorithm by van Groningen \& Wanders (1992) estimated errors in the scaled fluxes of better 
than 5\%, however, past studies employing somewhat improved versions of this same scaling algorithm typically achieved dispersions across a data set of $\sim 2 \%$ (e.g., Peterson et al. 1995). We measure a dispersion of $\sim 1.5 \%$, demonstrating the high level of homogeneity that we have been able to achieve in the current data set.

\subsection{Photometry}

In addition to spectral observations, we obtained supplemental $V$-band photometry from the 2.0-m Multicolor Active Galactic NUclei Monitoring (MAGNUM) telescope at the Haleakala Observatories in Hawaii, the 70-cm telescope of the CrAO, and the 0.4-m telescope of the University of Nebraska.

The MAGNUM observations were imaged with the multicolor imaging photometer (MIP) as described by Kobayashi et al. (1998a,b), Yoshii (2002), and Yoshii et al. (2003). Photometric fluxes measured from 23 observations between JD2454182 and JD2454311 within an aperture of 8".3. Photometric reduction of NGC 4051 was similar to that de-

scribed for other sources by Minezaki et al. (2004) and Suganuma et al. (2006), except the host-galaxy contribution to the flux within the aperture was not subtracted and the filter color term was not corrected because these photometric data were later scaled to the MDM continuum light curve (as described below). Also, minor corrections (of order 0.01 mag or less) due to the seeing dependence of the host-galaxy flux were ignored.

The 76 CrAO photometric observations were collected between J245D4180 and JD2454299 with the AP7p CCD mounted at the prime focus of the 70-cm telescope $(f=282 \mathrm{~cm})$. In this setup, the $512 \times 512$ pixels of the CCD field covers a 15 ! $\times 15$ ! field of view. Photometric fluxes were measured within an aperture of $15^{\prime \prime} 0$. For further details of the CrAO $V$-band observations and reduction, see the similar analysis described by Sergeev et al. (2005).

The University of Nebraska observations were conducted by taking and separately measuring a large number of one-minute images $(\sim 20)$ each of 28 nights between JD2454195 and JD2454290. Details of the observing and reduction procedure are as described by Klimek et al. (2004). Comparison star magnitudes were calibrated following Doroshenko et al. (2005a, b) and Chonis \& Gaskell (2008). To minimize the effects of variations in the image quality, fluxes were measured through an aperture of radius 8 arcseconds. The errors given for each night are the errors in the means. 


\subsection{Light Curves}

Light curves of the $\mathrm{H} \beta$ flux were made based on integrated fluxes measured in the MDM and CrAO spectra between 4815-4920 $\AA$ and over a linearly interpolated continuum defined between the average flux density in each of the following regions blueward and redward of $\mathrm{H} \beta$, respectively: $4770-4780 \AA$ and 5090-5130 $\AA$. The CrAO H $\beta$ light curve was then scaled to the MDM light curve with a multiplicative constant based on the average flux ratio between the four pairs of closely spaced points in the MDM and $\mathrm{CrAO} \mathrm{H} \beta$ light curves separated by no more than 0.5 day. This scaling is necessary to account for differences in the amount of [O III $]$ 5007 emission line flux that enters the slit in the different data sets due to seeing and aperture affects. The lower panel of Figure 2 shows the $\mathrm{H} \beta$ light curve derived from both data sets after scaling the CrAO fluxes to those measured from MDM spectra.

A continuum light curve was created with observations from each $V$-band photometric data set and the average continuum flux density measured over 5090-5130 $\AA$ (i.e., rest frame $\sim 5100 \AA$ ) in each spectrum of the spectroscopic data sets. First, the multiplicative scale factor determined for above to scale the $\mathrm{CrAO} \mathrm{H} \beta$ fluxes to the MDM light curve was also applied to the $\mathrm{CrAO}$ continuum fluxes, since the calibration of these fluxes is also susceptible to the same seeing and aperture affects as the $\mathrm{H} \beta$ flux calibration. Next, this light curve as well as the individual photometric light curves (see Fig. 2, upper panel) were scaled, one by one, to the same flux scale as the MDM light curve by making an additive, relative flux adjustment to each. This additive correction is necessary for both spectroscopic and photometric data because of differences in host galaxy starlight that enters the different aperture sizes of the various data sets. For the photometric observations, there is an additional component (also additive) due to the larger width of the filter bandpass. Each light curve was merged with the parent light curve to which it was scaled before the next light curve was scaled, thus building up a larger, more well-sampled light curve in the following order: MDM, MAGNUM, CrAO photometry, University of Nebraska, and CrAO spectroscopy. This was done so that the smaller, and in some cases shorter, light curves could be scaled to a longer and more densely sampled parent light curve. The scale factor applied to each secondary light curve to scale it to its parent light curve was calculated based on the difference between a linear least squares fit to this light curve and to the parent light curve before it, starting with the MDM light curve as the initial parent light curve. The fits to each light curve, both parent and secondary were limited to using only observations within the same overall temporal range, so that, when necessary, the beginning and/or ends of the light curves were truncated during the fitting process.

Assignment of uncertainties to the photometric fluxes is described above in the text or in references describing the photometric data sets. However, we calculated the uncertainties 
in the MDM and CrAO spectroscopic fluxes after creating the light curves for these data sets but before intercalibration. Typically, we determine uncertainties in our light curve flux measurements by applying a mean fractional error to all points. This fractional error is determined by comparing the average flux difference between closely spaced pairs of observations, assuming that flux differences across these short times scales are due to noise rather than genuine variability. Because real variability has been confirmed by Klimek et al. (2004) to occur in NGC 4051 on time scales shorter than 2 days, and our sampling rate is $\sim 1$ day, on average, we could not use this method to determine the relative errors on our spectroscopic flux measurements for this object. Instead, we took advantage of the observations of other higher-luminosity AGNs that we monitored as part of this larger campaign (i.e., same telescopes, instrumental setup, and observing conditions; results in preparation). Unlike NGC 4051, these objects neither exhibit variability on such short time scales nor have such short measured lags. Therefore, the uncertainties assigned to the NGC 4051 spectroscopic observations seen in Figure 2 are an average of the uncertainties in the flux measurements calculated as described above from closely spaced observations (separations of $\lesssim 2.0$ days) of these other objects (e.g., typical fractional errors in the range $\sim 0.12-0.21$ and $\sim 0.14-0.27$ for the continuum and line fluxes, respectively).

The merged continuum and $\mathrm{H} \beta$ light curves shown in Figure 3 are used for the subsequent time series analysis. These differ from simply combining the individual light curves, shown in Figure 2, in the following ways:

- First, we applied an absolute flux calibration to both light curves by applying a single multiplicative scale factor determined from the ratio of the [O III] $\lambda 5007$ emission line flux determined by P00 to that measured in the reference spectrum used above for the relative flux calibration. Unlike the emission line flux in our reference spectrum, the P00 flux measurement of $F([\mathrm{O} \mathrm{III}] \lambda 5007)=(3.91 \pm 0.12) \times 10^{-13} \mathrm{erg} \mathrm{s}^{-1} \mathrm{~cm}^{-2}$ was taken from observations obtained under photometric conditions and, consequently, $\sim 8 \%$ larger than our measured value. Additionally, the P00 measurement was made employing observing strategies and measurement practices similar to what we present here, thus validating this direct comparison. This additional flux calibration does not affect the reverberation results but is necessary for accurately measuring the $5100 \AA$ continuum luminosity.

- Second, we subtracted the host galaxy starlight contribution to the continuum flux, determined using the methods of Bentz et al. (2009a) to be $F_{\text {gal }}(5100 \AA)=(9.18 \pm$ $0.85) \times 10^{-15} \mathrm{erg} \mathrm{s}^{-1} \mathrm{~cm}^{-2} \AA^{-1}$.

- Third, we binned closely spaced observations as a weighted average and applied this 
to continuum flux measurements separated by $\leq 0.25$ days and $\mathrm{H} \beta$ flux measurements separated by $\leq 0.5$ days.

Fluxes for individual observations (i.e., before time binning) from all sources are listed in Table 1. Values listed represent the flux of each observation after completing all flux calibrations described above (i.e., relative calibration to intercalibrate all data sets onto the MDM flux scale, followed by absolute calibration based on the P00 [O III] $\lambda 5007$ line flux and removal of host starlight contamination). Column 1 gives the Julian Date of each observation. The $5100 \AA$ continuum or $V$-band flux and integrated $\mathrm{H} \beta$ flux are given in columns (2) and (3), respectively, and column (4) lists the source of each measurement. Photometric and spectroscopic observations from CrAO can be differentiated by noting that no $\mathrm{H} \beta$ flux values are present for photometric observations.

Table 2 displays statistical parameters describing the final light curves shown in Figure 3. Column (1) gives the spectral feature represented by each light curve, and the number of data points in each light curve is shown in column (2). Columns (3) and (4) are mean and median sampling intervals, respectively, between data points. The mean flux with standard deviation is given in column (5), while column (6) shows the mean fractional error in these fluxes. Column (7) gives the excess variance, calculated as

$$
F_{\mathrm{var}}=\frac{\sqrt{\sigma^{2}-\delta^{2}}}{\langle f\rangle}
$$

where $\sigma^{2}$ is the variance of the observed fluxes, $\delta^{2}$ is their mean square uncertainty, and $\langle f\rangle$ is the mean of the observed fluxes (Rodriguez-Pascual et al. 1997; Edelson et al. 2002). Finally, column (8) is the ratio of the maximum to minimum flux in the light curves.

\subsection{Time Series Analysis}

A times series analysis of the continuum and $\mathrm{H} \beta$ light curves was performed to determine the mean light travel time lag between continuum and emission line variations. We used two cross correlation schemes designed for data sets with uneven time sampling:

1. An interpolation scheme (Gaskell \& Sparke 1986; Gaskell \& Peterson 1987; White \& Peterson 1994) with an interval of 0.2 day. A cross-correlation function (CCF) is constructed from the mean value of the correlation coefficient, $r$, computed from cross correlating both the interpolated line light curve with the original continuum light curve and then 
the interpolated continuum light curve with the original line light curve, a process during which a range of possible lags, $\tau$, are imposed on the $\mathrm{H} \beta$ light curve.

2. A time binning scheme (Edelson \& Krolik 1988; White \& Peterson 1994) with a bin size of 1.0 day. Here, a discrete correlation function (DCF) is produced, which determines $r$ as a function of lag, similar to the CCF. In this scheme, however, only the actual data are cross correlated, and the resulting values of $r$ for all discretely correlated pairs are binned as a function of lag. The DCF that results gives the mean value of $r$ in each bin, where the corresponding uncertainty is assigned in a statistical manner (see White \& Peterson 1994). This method prevents possible spurious lag determinations that could potentially arise in the interpolation method due to under-sampling or large gaps in the data.

The resulting $\mathrm{CCF}$ and DCF are shown in Figure 4, along with the auto-correlation function $(\mathrm{ACF})$ computed by cross correlating the continuum with itself. We characterize the time delay between the continuum and emission line variations using two parameters derived from the CCF; $\tau_{\text {peak }}$ is the lag that corresponds to the largest correlation coefficient, $r_{\max }$, and $\tau_{\text {cent }}$ is the centroid of the CCF based on all points with $r \geq 0.8 r_{\max }$. Time dilation corrected values of $\tau_{\text {peak }}$ and $\tau_{\text {cent }}$ determined from the CCF in Figure 4 are given in Table 3. Uncertainties in both lag parameters are computed via model-independent Monte-Carlo simulations that employ the bootstrap method of Peterson et al. (1998), with the additional modifications of Peterson et al. (2004).

\section{Comparison with Previous Results}

Our measured $\mathrm{H} \beta$ lag of $\tau_{\text {cent }}=1.87_{-0.50}^{+0.54}$ days from this work is consistent, within the errors, to the most recent results for this object by S03, who measured a lag of $\tau_{\text {cent }}=3.1 \pm 1.6$ days. It is not clear how meaningful a direct comparison might be, however, because S03 measured the time delay between variations in the $\sim 6800 \AA$ continuum flux density and the integrated $\mathrm{H} \alpha$ flux rather than between the $\sim 5100 \AA$ continuum and $\mathrm{H} \beta$. We also note that the median sampling rate of S03 was larger than our measured lag, suggesting to us that the S03 light curves are undersampled. Furthermore, S03 only perform a cross correlation analysis based on the DCF method, which sacrifices time resolution.

Our new time delay measurements are inconsistent, however, with the previous measurement of $\tau_{\text {cent }}=5.8_{-1.8}^{+2.6}$ days by Peterson et al. (2004) using data from P00. These differences are unlikely a luminosity effect, since the average luminosity states of NGC 4051 were similar during this and the P00 campaigns $\left(\log \lambda L_{5100}=41.82\right.$ and $\log \lambda L_{5100}=41.87$, 
respectively)1. Therefore, we carefully re-examined the light curves used by P00 to better understand possible causes for the observed inconsistency.

Netzer \& Maoz (1990) suggested that the cause for a similar inconsistency between lag measurements from two reverberation mapping campaigns of NGC 5548 (Netzer et al. 1990; Peterson et al. 1991) was due to different continuum variability timescales observed in the separate campaigns: longer continuum variability timescales lead to larger lag measurements. However, this explanation is unlikely to be the cause for the current inconsistency between our measured lag and that of P00 because the prominent variability timescales observed in both the P00 and current continuum light curves are similar ( $\sim 40-50$ days). Instead, the simplest explanation for the inconsistency between our measured lag and that of P00 is random error. We investigated this possibility by performing Monte Carlo simulations using the "Subset $1 " \mathrm{H} \beta$ and $5100 \AA$ continuum flux light curves from P00 with the goal of estimating the likelihood that a lag of $\tau_{c e n t}=5.8$ days would be measured, even if the actual BLR radius of the $\mathrm{H} \beta$ emission was 2.7 light days, as expected from the Bentz et al. (2009a) $R-L$ relation for the average luminosity of NGC 4051 during this time period. In each simulation we created a simulated $\mathrm{H} \beta$ light curve by convolving a modified continuum light curve with a transfer function that assumed a BLR with a thin spherical shell geometry of radius 2.7 light days. The sampling was increased in the modified continuum light curve over that of the original Subset 1 continuum light curve by interpolating between the actual points on a 0.5 day scale. Noise was added to the flux of each interpolated point using a random Gaussian deviate. The size of each deviate was based on the average uncertainty in flux of the closest 'real' continuum point on each side of the interpolated point. The simulated emission-line light curve was then sampled identically to the original Subset $1 \mathrm{H} \beta$ light curve. We cross correlated this new emission-line light curve with the original Subset 1 continuum light curve to determine a reverberation lag. The simulation was repeated 10,000 times, and lags were measured similarly for each iteration. We found that the average lag recovered was 2.7 days (reassuring, since this was our input radius). However, we were unable to reproduce even a single lag of 5.8 days. In fact, the largest lag our simulations recovered was 4.0 days. A couple of possibilites suggest themselves:

- The BLR has physically changed in the 11-year interval between the time of P00's Subset 1 and the time of our recent campaign. This is a physical possibility since the dynamical timescale of the BLR in NGC 4051 is $\lesssim 5$ years.

- The P00 data are undersampled and there are really unresolved variations occurring

\footnotetext{
${ }^{1}$ The average observed flux of the S03 campaign was within $\sim 10 \%$ that of the Peterson et al. campaign as well.
} 
on timescales shorter than the typical sampling interval of 2.2 days in Subset 1, the best-sampled part of the P00 light curve.

We have no particular reason to believe the former possibility. However, the latter is suggested by how P00 established the relative uncertainties of their fluxes, namely by assuming that there are no true variations on time scales shorter that the typical sampling time scales and that any differences between closely spaced measurements reflect random errors only, not true variability. The estimates of the relative flux errors in the P00 Subset 1 based on comparing measurements separated by 2 days or less are about $3.2 \%$ for both the contiuum and the line. In our new data set, obtained with the same instrument, we find relative errors of about $1.4 \%$ and $2.1 \%$ in the continuum and the line, respectively, using the method described in Section 2.3. We conclude that the flux uncertainties of the P00 data were overestimated due to short timescale variability.

Proceeding with the assumption that the P00 light curves are undersampled, we isolated the portion of the light curves that has the highest sampling across the sharpest features. We made this selection in an attempt to avoid occurrences of undersampling more complex variability. From the initial light curve, reproduced in Figure 5, we removed the first 10 observations that exhibit a broad inflection in the flux with a poorly defined peak. We perform a cross correlation analysis on these shortened light curves and determine a shorter lag, $\tau_{\text {cent }}=3.5_{-1.9}^{+3.7}$ days. This lag determination is consistent with both the expected radius of 2.7 light days from the $R-L$ relation, the current results, and the results of S03.

If we continue with the assumption that the light curves of P00 are undersampled, and the P00 flux uncertainties are overestimated, their assigned uncertainties would act to decrease the significance of short timescale variability, likely attributing it to noise instead. If we impose the average continuum flux uncertainty measured from our current data set (given above) on the shortened P00 continuum light curve, we can further improve the precision of this revised lag measurement to $\tau_{\text {cent }}=3.5_{-1.5}^{+3.2}$ days.

We then conducted another simulation in which we applied the sampling rate from the P00 light curves to the light curves from this work. By undersampling our current light curves, we can determine the probability that undersampling could lead to an overestimated lag similar to that measured by P00. This type of simulation can provide further evidence that the lag measured by P00 was an overestimate and a consequence of undersampling. At the same time, it could diminish the possibility that the discrepancy in lag measurements is due to a difference in the physical conditions or structure of the BLR during the P00 campaign compared to the present. Using the continuum and $\mathrm{H} \beta$ light curves shown in Figure 3 as the starting point, we modified them similarly to the continuum light curve described for our first set of simulations (i.e., increasing the sampling by interpolating between data points 
and adding noise to these points with a Gaussian deviate), but this time we interpolated both the continuum and emission line light curves from this work on a 0.1 day interval. We then drew sample light curves from this parent light curve with the same length and sampling pattern as the full P00 light curve shown in Figure 5. We applied the same cross-correlation analysis (as described in Section 2.4) to measure lags from these sample light curves. The parent light curves cover a longer time span than the sample light curves and therefore allow for multiple iterations of sample light curves to be chosen from different subsets of the parent light curves. The first iteration of sample light curves are created from the subset of the parent light curves where the beginning points match up, but the ends of the parent light curves are discarded. We then build up multiple iterations by shifting the starting point of the sample light curve in time by one time step, i.e., 0.1 day, across the parent light curves. In this way, we were able to build up 330 sample light curves, where in the last iteration, the sample light curves begin in the middle of the parent light curves, but both sets of light curves end at the same time. Based on the cross correlation analysis from these 330 sample light curves, the probability of measuring $\tau_{\text {cent }} \geq 5.0$ days is $0.6 \%$ ( 2 out of 330 ), and the probability of measuring $\tau_{\text {cent }} \geq 3.5$ days (i.e, the lag we calculated above from only a portion of the P00 light curve) is $\sim 8 \%$ (25 out of 330). We conclude that undersampling is at least a plausible explanation for the difference between the P00 results and those reported here.

\section{Black Hole Mass}

Applying virial assumptions to the reverberating gas in the BLR, the mass of the black hole can be defined by

$$
M_{\mathrm{BH}}=\frac{f c \tau(\Delta V)^{2}}{G}
$$

where $\tau$ is the measured emission-line time delay, so that $\mathrm{c} \tau$ represents the BLR radius, and $\Delta V$ is the BLR velocity dispersion (Peterson et al. 2004). The dimensionless factor $f$ depends on the structure, kinematics, and inclination of the BLR and is of order unity.

We estimate the BLR velocity dispersion from the line width of $\mathrm{H} \beta$ emission line. The line width can be characterized by either the FWHM or the line dispersion, i.e., the second moment of the line profile. The FWHM and the line dispersion, $\sigma_{\text {line }}$, were measured from both the mean and the rms spectra of NGC 4051 shown in Figure 1, Here, we have measured both quantities and their uncertainties employing methods described in detail by Peterson et al. (2004). All measured values of the $\mathrm{H} \beta$ line width are listed in Table 3 . Typically, the narrow-line emission component of the line should be removed before measuring 
the line width (see Denney et al. 2009); however, this component could not be reliably isolated from the rest of the line profile in this object. As a result the line widths measured in the mean spectrum, particularly the FWHM, are less reliable for these purposes than the widths measured from the rms spectrum 2 .

We calculate the black hole mass for NGC 4051 using $\tau_{\text {cent }}$, for the time delay, $\tau$, and the line dispersion, $\sigma_{\text {line }}$, measured from the $\mathrm{H} \beta$ emission line in the rms spectrum, for the emission-line width, $\Delta V$. We utilize the calibration of the reverberation mass scale of Onken et al. (2004) for this choice of lag and line width parameters and therefore adopt a scale factor value of $f=5.5$. We then use equation 2 to estimate the black hole mass of NGC 4051 to be $M_{\mathrm{BH}}=\left(1.73_{-0.52}^{+0.55}\right) \times 10^{6} M_{\odot}$. Here, statistical and observational uncertainties have been included, but intrinsic uncertainties from sources such as unknown BLR inclination cannot be accurately ascertained.

Marconi et al. (2008) have considered the effect of radiation pressure on SMBH mass estimates and provide a new prescription for calculating the SMBH mass that includes a correction factor to account for radiation pressure. Radiation pressure acts to partially counteract the force of gravity on the BLR gas, since the outward radiation force has the same radial dependence $\left(r^{-2}\right)$ as the inward gravitational force. As a result the BLR gas motions are effectively under the influence of an apparently smaller SMBH mass, which leads to an underestimate of the true SMBH mass. Marconi et al. (2008) determine that although taking radiation pressure into account is most important for black holes radiating near the Eddington limit, it should be considered even for systems with $L<L_{\mathrm{Edd}}$. On the other hand, in a comparison study of Type 1 versus Type 2 black hole masses determined with independent methods, Netzer (2009) sees better agreement between the mass and $L / L_{\text {Edd }}$ distributions of these two populations if radiation pressure forces are neglected. Netzer concludes that either the effects of radiation pressure on the BLR gas in these objects is negligible or that BLR column densities must be significantly larger, i.e., $N_{\mathrm{H}} \gtrsim 10^{24} \mathrm{~cm}^{-2}$, than assumed by Marconi et al. $\left(2008 ; N_{\mathrm{H}}=10^{23} \mathrm{~cm}^{-2}\right)$. In a more recent paper, Marconi et al. (2009) reinvestigate the results of Netzer (2009) and support their findings on the dependence of the effect of radiation pressure on column density but conclude that, until it is possible to determine the nature of the apparent dependence of $N_{\mathrm{H}}$ on source properties (e.g., $L / L_{\mathrm{Edd}}$ ), one should always consider the possibility that radiation forces are important, and black hole masses should consequently be determined using the correction of Marconi et al. (2008).

\footnotetext{
${ }^{2}$ Since only BLR emission varies in response to the ionizing continuum on reverberation timescales, flux contributions from the narrow-line component will not contaminate the line width measurement in the rms spectrum.
} 
The importance of radiation pressure forces on black hole mass determinations is still under debate. Therefore, in addition to our virial mass estimate for NGC 4051 given above, we also estimate the SMBH mass in NGC 4051 taking radiation pressure into consideration (cf. equation 6 of Marconi et al. 2008), with $f=3.1 \pm 1.4$ and $\log g=7.6 \pm 0.3$, which are derived by Marconi et al. from fits to SMBH masses from reverberation mapping studies. With these scale factors and the same line width and BLR radius measurements used above, we calculate a mass of $M_{\mathrm{BH}-\mathrm{rad}}=\left(1.24_{-0.56}^{+0.57}\right) \times 10^{6} M_{\odot}$. Contrary to the expectations from the physical arguments, we calculate a mass smaller than that determined in the case where we did not consider the effect of radiation pressure. Because NGC 4051 is a low-luminosity AGN radiating well below the Eddington limit $\left(L / L_{\mathrm{Edd}}=0.030\right)$, the correction to the mass due to radiation pressure is small (smaller even than the formal observational uncertainties on the mass), adding only $0.26 \times 10^{6} M_{\odot}$ to the mass. However, this radiation-corrected mass estimate is smaller than our original estimate because the value of $f$ derived by Marconi et al. is a factor of 1.8 smaller than the Onken et al. (2004) value we adopted above. Because this scale factor was derived in a statistical sense by both Onken et al. and Marconi et al., the difference in $f$ values between these two studies has the potential to affect the mass of an individual object more than would be expected for a statistical sample, particularly for the low accretion-rate objects that need only a small radiation pressure correction.

\section{Velocity-Resolved Investigation}

The lag measurements between the continuum and $\mathrm{H} \beta$ emission in Table 3 represent the average time delay across the BLR. Because the BLR is an extended region and the velocity of the gas is most likely a function of position, gas in different locations of the BLR should respond to variations in the ionizing continuum flux on slightly different time scales. The observable result should be a difference in the reverberation lag measurement in different parts of the line profile (i.e., separated in velocity space). Velocity-resolved reverberation mapping thus gives us information about the kinematics of gas in the BLR. Previous studies of time delay differences between multiple emission lines and the velocity dependence of the lag within a single emission line have shown that the BLR is virialized and commonly contains an additional inflow component (e.g., Gaskell 1988; Koratkar \& Gaskell 1991; Korista et al. 1995; Done \& Krolik 1996; Welsh et al. 2007; Bentz et al. 2008); however, the creation of full velocity-delay maps (see Horne et al. 2004) is an aspect of the reverberation mapping technique that has not yet been fully realized (though see Horne et al. 1991, Done \& Krolik 1996, Ulrich \& Horne 1996, and Kollatschny 2003 for previous attempts). By resolving the velocity-dependent reverberation response of the BLR better than has been done in the past, we can reconstruct and analyze the velocity-delay map to gain further insights into 
the geometry and kinematics of the BLR.

We searched for a velocity-dependent reverberation signal by dividing the $\mathrm{H} \beta$ emission line flux into 8 velocity-space bins. The blue and red sides of the line were separately divided into 4 bins of equal velocity width, covering only the most variable portions of the line profile, i.e., the outer-most wavelength boundaries were reduced from those considered for the analysis of the full profile to only include flux within the range 4840-4900^ (roughly $\left.\pm 2,000 \mathrm{~km} \mathrm{~s}^{-1}\right)$. Light curves were created from measurements of the integrated $\mathrm{H} \beta$ flux in each bin and then cross correlated with the continuum light curve following the same procedures described above. The top panel of Figure 6 shows the division of the $\mathrm{H} \beta$ line profile from the rms spectrum into the eight velocity bins, and the bottom panel shows the lag measurements for each of these bins. Error bars in the velocity direction represent the bin width. The evidence for a velocity-stratified BLR response to continuum variations is present, but marginal. In particular, the lags measured for bins 1-2 and 7-8 are consistent with zero and might simply reflect correlated errors due to continuum contamination. Although the shape of the velocity-resolved signal in Figure 6 supports our virial assumptions, since the higher velocity gas varies on shorter time scales than the low velocity gas, there are no strong indications for either outflow or inflow. Outflow could be suggested by the larger lag measured in bin 5 (red side of the line) compared to bin 4 (blue side of the line), but the difference is very marginal. Even with the high sampling rate and spectral resolution we achieved during this campaign, observing a velocity-resolved signal for NGC 4051 as clearly as that detected for Arp 151 by Bentz et al. (2008) would have been rather surprising for the following reasons. First, the precision with which we can measure a lag is somewhat dependent on the median observational sampling interval, which, for NGC 4051, was still not much shorter than the measured lag. This indicates that in order to better resolve a velocitydependent signal, we need even higher time resolution for this object. Second, because the $\mathrm{H} \beta$ line is particularly narrow in this object, there are only a few velocity resolution elements across the line.

\section{Discussion}

Based on our simulations, reanalysis of the light curves from P00, and the additional arguments we presented above, we conclude that the inconsistency between the past measurements of the $\mathrm{H} \beta$ reverberation lag in NGC 4051 and our present measurements most likely results from an overestimation of the lag by $\mathrm{P} 00$. Therefore, we adopt the results from the current reverberation campaign over previous campaign results measuring this lag in NGC 4051. Using the lag measurement of $\tau_{c e n t}=1.87_{-0.50}^{+0.54}$ days presented here and the 
Tully-Fisher distance of Russell (2003), NGC 4051 is no longer an outlier on the $R_{\mathrm{BLR}}-L$ relationship. Figure 7 replicates the most recent version of this relationship by Bentz et al. (2009a) with both the previous and current lag values of NGC 4051 marked. Secure placement of low-luminosity objects such as NGC 4051 on the $R_{\mathrm{BLR}}-L$ relationship is important for supporting the extrapolation of this relationship to the even lower-luminosity regime potentially populated by intermediate-mass black holes. Additional results from the present campaign (Denney et al., in preparation), as well as results from a recent monitoring campaign at the Lick Observatory (e.g., Bentz et al. 2008), aim to further populate this lowluminosity end of the $R_{\mathrm{BLR}}-L$ relationship, thus solidifying the calibration in a relatively under-sampled region of the relation. A reliable calibration of this relationship is imperative for large studies of black hole masses and galaxy evolution, since it allows for the calculation of black hole masses from single-epoch spectra and provides luminosity and radius estimates that help constrain parameter space in the search for intermediate-mass black holes.

Our new results provide a measure of the BLR radius of NGC 4051 that is closer to the value naively expected from its luminosity. However, these new results do not resolve the unexpected location of this object on the $M_{\mathrm{BH}}-L$ relation: NLS1s tend to lie on a locus of this relation with relatively high Eddington ratios $\left(L / L_{\text {Edd }} \gtrsim 0.1\right.$; see Figure 16 of Peterson et al. 2004). However, based on the black hole mass of $M_{\mathrm{BH}}=\left(1.73_{-0.52}^{+0.55}\right) \times 10^{6} M_{\odot}$ that we have calculated, the Eddington ratio of NGC 4051 is still only $L / L_{\mathrm{Edd}}=0.030$. It seems unlikely that we have overestimated the mass of the black hole (and thus underestimated $L / L_{\text {Edd }}$ ), since our mass measurement is already a factor of a few lower than predicted by the $M_{\mathrm{BH}}-\sigma_{\star}$ relationship (Nelson \& Whittle 1995; Ferrarese et al. 2001). In this case, the narrowness of the Balmer lines in the spectrum of NGC 4051 might be due at least in part to the inclination of the BLR relative to our line of sight — indeed, inclination has been invoked as one possible way to explain the NLS1 phenomenon since the early days of research on these sources (e.g., Osterbrock \& Pogge 1985; Boller et al. 1996). From observations of the narrow-line region in NGC 4051, Christopoulou et al. (1997) estimate that the inclination of this source is $\sim 50 \mathrm{deg}$, near the maximum expected for a Type 1 active nucleus in unified models. It is entirely reasonable to suppose that the BLR and accretion disk are at the same inclination as the narrow-line region. Even so, accounting for this high inclination would increase the line width by only about a factor of 2, still within the NLS1 regime. Clearly further investigation is required to understand the low value of $L / L_{\mathrm{Edd}}$ in NGC 4051 compared to other NLS1s.

We would like to thank the anonymous referee for useful suggestions that improved the clarity of this manuscript. We acknowledge support for this work by the National Science Foundation though grant AST-0604066 to The Ohio State University. CMG is grateful for support by the National Science Foundation through grants AST 03-07912 and AST 
08-03883. MV acknowledges financial support from HST grants HST-GO-10417, HST-AR10691, and HST-GO-10833 awarded by the Space Telescope Science Institute, which is operated by the Association of Universities for Research in Astronomy, Inc., for NASA, under contract NAS5-26555. VTD acknoledges the support of the Russian Foundation for Basic Research (project no. 06-02-16843) to the Crimean Laboratory of the Sternberg Astronomical Institute. SGS acknowledges support through Grant No. 5-20 of the "Cosmomicrophysics" program of the National Academy of Sciences of Ukraine to CrAO. The CrAO CCD cameras have been purchased through the US Civilian Research and Development Foundation for the Independent States of the Former Soviet Union (CRDF) awards UP1-2116 and UP12549-CR-03. This research has made use of the NASA/IPAC Extragalactic Database (NED) which is operated by the Jet Propulsion Laboratory, California Institute of Technology, under contract with the National Aeronautics and Space Administration. 


\section{REFERENCES}

Bennert, N., Canalizo, G., Jungwiert, B., Stockton, A., Schweizer, F., Peng, C. Y., \& Lacy, M. 2008, ApJ, 677, 846

Bentz, M. C., Peterson, B. M., Netzer, H., Pogge, R. W., \& Vestergaard, M. 2009a, ApJ, 697,160

Bentz, M. C., Peterson, B. M., Pogge, R. W., \& Vestergaard, M. 2009b, ApJ, 694, L166

Bentz, M. C., Peterson, B. M., Pogge, R. W., Vestergaard, M., \& Onken, C. A. 2006, ApJ, 644,133

Bentz, M. C., et al. 2008, ApJ, 689, L21

Blandford, R. D., \& McKee, C. F. 1982, ApJ, 255, 419

Boller, T., Brandt, W. N., \& Fink, H. 1996, A\&A, 305, 53

Chonis, T. S., \& Gaskell, C. M. 2008, AJ, 135, 264

Christopoulou, P. E., Holloway, A. J., Steffen, W., Mundell, C. G., Thean, A. H. C., Goudis, C. D., Meaburn, J., \& Pedlar, A. 1997, MNRAS, 284, 385

Corbett, E. A., et al. 2003, MNRAS, 343, 705

Denney, K. D., Peterson, B. M., Dietrich, M., Vestergaard, M., \& Bentz, M. C. 2009, ApJ, 692,246

Denney, K. D., et al. 2006, ApJ, 653, 152

Done, C., \& Krolik, J. H. 1996, ApJ, 463, 144

Doroshenko, V. T., Sergeev, S. G., Merkulova, N. I., Sergeeva, E. A., Golubinsky, Y. V., Pronik, V. I., \& Okhmat, N. N. 2005a, Astrophysics, 48, 156

—. 2005b, Astrophysics, 48, 304

Edelson, R., Turner, T. J., Pounds, K., Vaughan, S., Markowitz, A., Marshall, H., Dobbie, P., \& Warwick, R. 2002, ApJ, 568, 610

Edelson, R. A., \& Krolik, J. H. 1988, ApJ, 333, 646

Ferrarese, L., \& Merritt, D. 2000, ApJ, 539, L9 
Ferrarese, L., Pogge, R. W., Peterson, B. M., Merritt, D., Wandel, A., \& Joseph, C. L. 2001, ApJ, 555, L79

Fine, S., et al. 2008, MNRAS, 390, 1413

Gaskell, C. M. 1988, ApJ, 325, 114

Gaskell, C. M., \& Peterson, B. M. 1987, ApJS, 65, 1

Gaskell, C. M., \& Sparke, L. S. 1986, ApJ, 305, 175

Gebhardt, K., et al. 2000a, ApJ, 539, L13

—. 2000b, ApJ, 543, L5

Graham, A. W. 2007, MNRAS, 379, 711

Hopkins, P. F., \& Hernquist, L. 2009, ApJ, 694, 599

Horne, K., Peterson, B. M., Collier, S. J., \& Netzer, H. 2004, PASP, 116, 465

Horne, K., Welsh, W. F., \& Peterson, B. M. 1991, ApJ, 367, L5

Kaspi, S., Maoz, D., Netzer, H., Peterson, B. M., Vestergaard, M., \& Jannuzi, B. T. 2005, ApJ, 629, 61

Kaspi, S., Smith, P. S., Netzer, H., Maoz, D., Jannuzi, B. T., \& Giveon, U. 2000, ApJ, 533, 631

Klimek, E. S., Gaskell, C. M., \& Hedrick, C. H. 2004, ApJ, 609, 69

Kobayashi, Y., Yoshii, Y., Peterson, B. A., Minezaki, T., Enya, K., Suganuma, M., \& Yamamuro, T. 1998a, in Proc. SPIE, Vol. 3354, 769-776

Kobayashi, Y., et al. 1998b, in Proc. SPIE, Vol. 3352, 120-128

Kollatschny, W. 2003, A\&A, 407, 461

Kollmeier, J. A., et al. 2006, ApJ, 648, 128

Koratkar, A. P., \& Gaskell, C. M. 1991, ApJ, 375, 85

Korista, K. T., et al. 1995, ApJS, 97, 285

Kormendy, J., \& Richstone, D. 1995, ARA\&A, 33, 581 
Magorrian, J., et al. 1998, AJ, 115, 2285

Marconi, A., Axon, D. J., Maiolino, R., Nagao, T., Pastorini, G., Pietrini, P., Robinson, A., \& Torricelli, G. 2008, ApJ, 678, 693

Marconi, A., Axon, D. J., Maiolino, R., Nagao, T., Pietrini, P., Risaliti, G., Robinson, A., \& Torricelli, G. 2009, ApJ, 698, L103

Minezaki, T., Yoshii, Y., Kobayashi, Y., Enya, K., Suganuma, M., Tomita, H., Aoki, T., \& Peterson, B. A. 2004, ApJ, 600, L35

Nelson, C. H., Green, R. F., Bower, G., Gebhardt, K., \& Weistrop, D. 2004, ApJ, 615, 652

Nelson, C. H., \& Whittle, M. 1995, ApJS, 99, 67

Netzer, H. 2009, ApJ, 695, 793

Netzer, H., \& Maoz, D. 1990, ApJ, 365, L5

Netzer, H., Maoz, D., Laor, A., Mendelson, H., Brosch, N., Leibowitz, E., Almoznino, E., Beck, S., \& Mazeh, T. 1990, ApJ, 353, 108

Onken, C. A., Ferrarese, L., Merritt, D., Peterson, B. M., Pogge, R. W., Vestergaard, M., \& Wandel, A. 2004, ApJ, 615, 645

Osterbrock, D. E., \& Pogge, R. W. 1985, ApJ, 297, 166

Peterson, B. M. 1993, PASP, 105, 247

-. 2001, in Advanced Lectures on the Starburst-AGN Connection, ed. I. Aretxaga, D. (Singapore: World Scientific), 3

Peterson, B. M., Pogge, R. W., Wanders, I., Smith, S. M., \& Romanishin, W. 1995, PASP, 107,579

Peterson, B. M., Wanders, I., Bertram, R., Hunley, J. F., Pogge, R. W., \& Wagner, R. M. 1998, ApJ, 501, 82

Peterson, B. M., et al. 1991, ApJ, 368, 119

-. 2000, ApJ, 542, 161

-. 2004, ApJ, 613, 682

Rodriguez-Pascual, P. M., et al. 1997, ApJS, 110, 9 
Russell, D. G. 2003, astro-ph/0310284

Sergeev, S. G., Doroshenko, V. T., Golubinskiy, Y. V., Merkulova, N. I., \& Sergeeva, E. A. 2005, ApJ, 622, 129

Shankar, F., Weinberg, D. H., \& Miralda-Escudé, J. 2009, ApJ, 690, 20

Shemmer, O., Uttley, P., Netzer, H., \& McHardy, I. M. 2003, MNRAS, 343, 1341

Shen, J., Vanden Berk, D. E., Schneider, D. P., \& Hall, P. B. 2008a, AJ, 135, 928

Shen, Y., Greene, J. E., Strauss, M. A., Richards, G. T., \& Schneider, D. P. 2008b, ApJ, 680,169

Somerville, R. S., Hopkins, P. F., Cox, T. J., Robertson, B. E., \& Hernquist, L. 2008, MNRAS, 391, 481

Suganuma, M., et al. 2006, ApJ, 639, 46

Tremaine, S., et al. 2002, ApJ, 574, 740

Ulrich, M.-H., \& Horne, K. 1996, MNRAS, 283, 748

van Groningen, E., \& Wanders, I. 1992, PASP, 104, 700

Vestergaard, M. 2002, ApJ, 571, 733

—. 2004, ApJ, 601, 676

Vestergaard, M., Fan, X., Tremonti, C. A., Osmer, P. S., \& Richards, G. T. 2008, ApJ, 674, L1

Wandel, A. 2002, ApJ, 565, 762

Welsh, W. F., Martino, D. L., Kawaguchi, G., \& Kollatschny, W. 2007, in Astronomical Society of the Pacific Conference Series, Vol. 373, The Central Engine of Active Galactic Nuclei, ed. L. C. Ho \& J.-W. Wang, 29-+

White, R. J., \& Peterson, B. M. 1994, PASP, 106, 879

Yoshii, Y. 2002, in New Trends in Theoretical and Observational Cosmology, ed. K. Sato \& T. Shiromizu (Tokyo: Universal Academy), 235

Yoshii, Y., Kobayashi, Y., \& Minezaki, T. 2003, American Astronomical Society Meeting Abstracts, 202, 3803 
This preprint was prepared with the AAS IATEX macros v5.2. 
Table 1. $\quad V$-band, Continuum, and $\mathrm{H} \beta$ Fluxes for NGC 4051

\begin{tabular}{|c|c|c|c|}
\hline $\begin{array}{c}\text { JD } \\
(-2450000)\end{array}$ & $\begin{array}{c}F_{\lambda}(5100 \AA)^{\mathrm{a}} \\
\left(10^{-15} \operatorname{erg~s}^{-1} \mathrm{~cm}^{-2} \AA^{-1}\right)\end{array}$ & $\begin{array}{c}\mathrm{H} \beta \lambda 4861 \\
\left(10^{-13} \mathrm{erg} \mathrm{s}^{-1} \mathrm{~cm}^{-2}\right)\end{array}$ & Observatory \\
\hline 4180.30 & $5.03 \pm 0.18$ & $\ldots$ & $\mathrm{CrAO}$ \\
\hline 4181.37 & $5.66 \pm 0.16$ & $\ldots$ & $\mathrm{CrAO}$ \\
\hline 4182.02 & $5.47 \pm 0.09$ & $\ldots$ & MAGNUM \\
\hline 4182.41 & $5.07 \pm 0.22$ & $\ldots$ & $\mathrm{CrAO}$ \\
\hline 4184.79 & $5.41 \pm 0.20$ & $5.22 \pm 0.11$ & MDM \\
\hline 4185.71 & $4.51 \pm 0.19$ & $4.96 \pm 0.10$ & MDM \\
\hline 4186.49 & $4.51 \pm 0.22$ & $\ldots$ & $\mathrm{CrAO}$ \\
\hline 4186.71 & $4.89 \pm 0.20$ & $4.15 \pm 0.09$ & MDM \\
\hline 4187.38 & $4.82 \pm 0.22$ & . & $\mathrm{CrAO}$ \\
\hline 4187.86 & $5.27 \pm 0.20$ & $4.95 \pm 0.10$ & MDM \\
\hline 4188.37 & $4.32 \pm 0.25$ & $\ldots$ & $\mathrm{CrAO}$ \\
\hline 4188.71 & $4.81 \pm 0.20$ & $4.90 \pm 0.10$ & MDM \\
\hline 4189.39 & $4.75 \pm 0.26$ & $\ldots$ & $\mathrm{CrAO}$ \\
\hline 4189.61 & $4.78 \pm 0.19$ & $4.62 \pm 0.10$ & MDM \\
\hline 4189.96 & $5.53 \pm 0.21$ & 4.88ata & MAGNUM \\
\hline 4189.96 & $4.94 \pm 0.05$ & $0.00 \pm 0.10$ & $\mathrm{MDM}$ \\
\hline 4190.41 & $4.82 \pm 0.23$ & & $\mathrm{CrAO}$ \\
\hline 4190.72 & $4.82 \pm 0.20$ & $4.73 \pm 0.10$ & MDM \\
\hline 4191.38 & $4.59 \pm 0.32$ & $\ldots$ & $\mathrm{CrAO}$ \\
\hline 4191.62 & $4.91 \pm 0.20$ & $4.51 \pm 0.09$ & MDM \\
\hline 4191.91 & $5.27 \pm 0.20$ & $4.77 \pm 0.10$ & MDM \\
\hline 4192.43 & $4.84 \pm 0.32$ & $\ldots$ & $\mathrm{CrAO}$ \\
\hline 4192.75 & $4.02 \pm 0.19$ & $4.44 \pm 0.09$ & MDM \\
\hline 4193.61 & $4.86 \pm 0.20$ & $4.34 \pm 0.09$ & MDM \\
\hline 4193.92 & $4.41 \pm 0.19$ & $4.57 \pm 0.10$ & MDM \\
\hline 4194.73 & $4.62 \pm 0.19$ & $4.60 \pm 0.10$ & MDM \\
\hline 4195.45 & $4.49 \pm 0.16$ & $\ldots$ & UNebr. \\
\hline 4195.63 & $4.30 \pm 0.19$ & $4.06 \pm 0.09$ & MDM \\
\hline 4196.62 & $4.59 \pm 0.19$ & $4.22 \pm 0.09$ & MDM \\
\hline 4197.78 & $4.22 \pm 0.19$ & $4.43 \pm 0.09$ & MDM \\
\hline
\end{tabular}


Table 1-Continued

\begin{tabular}{|c|c|c|c|}
\hline $\begin{array}{c}\text { JD } \\
(-2450000)\end{array}$ & $\begin{array}{c}F_{\lambda}(5100 \AA)^{\mathrm{a}} \\
\left(10^{-15} \mathrm{erg} \mathrm{s}^{-1} \mathrm{~cm}^{-2} \AA^{-1}\right)\end{array}$ & $\begin{array}{c}\mathrm{H} \beta \lambda 4861 \\
\left(10^{-13} \mathrm{erg} \mathrm{s}^{-1} \mathrm{~cm}^{-2}\right)\end{array}$ & Observatory \\
\hline 4198.44 & $4.34 \pm 0.14$ & $\ldots$ & UNebr. \\
\hline 4198.77 & $4.51 \pm 0.19$ & $4.80 \pm 0.10$ & MDM \\
\hline 4198.93 & $4.46 \pm 0.06$ & $\ldots$ & MAGNUM \\
\hline 4199.36 & $4.68 \pm 0.17$ & $\ldots$ & $\mathrm{CrAO}$ \\
\hline 4199.40 & $4.78 \pm 0.15$ & $\ldots$ & UNebr. \\
\hline 4199.86 & $4.55 \pm 0.19$ & $4.37 \pm 0.09$ & MDM \\
\hline 4200.40 & $4.52 \pm 0.15$ & $\ldots$ & $\mathrm{CrAO}$ \\
\hline 4200.72 & $4.19 \pm 0.19$ & $4.29 \pm 0.09$ & MDM \\
\hline 4201.31 & $4.84 \pm 0.23$ & $\ldots$ & $\mathrm{CrAO}$ \\
\hline 4201.73 & $4.59 \pm 0.19$ & $4.49 \pm 0.09$ & MDM \\
\hline 4202.37 & $4.56 \pm 0.18$ & $\ldots$ & $\mathrm{CrAO}$ \\
\hline 4202.95 & $4.93 \pm 0.08$ & $\ldots$ & MAGNUM \\
\hline 4204.40 & $4.53 \pm 0.17$ & $\ldots$ & $\mathrm{CrAO}$ \\
\hline 4204.73 & $4.35 \pm 0.19$ & $4.58 \pm 0.10$ & MDM \\
\hline 4205.34 & $4.34 \pm 0.16$ & $\ldots$ & $\mathrm{CrAO}$ \\
\hline 4205.62 & $3.97 \pm 0.18$ & $4.37 \pm 0.09$ & MDM \\
\hline 4205.83 & $4.32 \pm 0.03$ & $\ldots$ & MAGNUM \\
\hline 4205.91 & $4.28 \pm 0.19$ & $4.54 \pm 0.10$ & MDM \\
\hline 4206.36 & $4.19 \pm 0.18$ & $\ldots$ & $\mathrm{CrAO}$ \\
\hline 4206.40 & $4.23 \pm 0.23$ & $\ldots$ & UNebr. \\
\hline 4206.62 & $4.31 \pm 0.19$ & $4.46 \pm 0.09$ & MDM \\
\hline 4207.40 & $4.30 \pm 0.15$ & $\ldots$ & UNebr. \\
\hline 4207.82 & $4.40 \pm 0.19$ & $4.60 \pm 0.10$ & MDM \\
\hline 4208.34 & $4.42 \pm 0.15$ & $\ldots$ & $\mathrm{CrAO}$ \\
\hline 4208.40 & $4.26 \pm 0.17$ & $\ldots$ & UNebr. \\
\hline 4208.63 & $4.24 \pm 0.19$ & $4.58 \pm 0.10$ & MDM \\
\hline 4208.88 & $4.25 \pm 0.03$ & $\ldots$ & MAGNUM \\
\hline 4209.40 & $4.46 \pm 0.15$ & $\ldots$ & $\mathrm{CrAO}$ \\
\hline 4209.78 & $4.81 \pm 0.20$ & $4.81 \pm 0.10$ & MDM \\
\hline 4210.62 & $5.01 \pm 0.20$ & $4.99 \pm 0.10$ & MDM \\
\hline
\end{tabular}


Table 1-Continued

\begin{tabular}{|c|c|c|c|}
\hline $\begin{array}{c}\text { JD } \\
(-2450000)\end{array}$ & $\begin{array}{c}F_{\lambda}(5100 \AA)^{\mathrm{a}} \\
\left(10^{-15} \mathrm{erg} \mathrm{s}^{-1} \mathrm{~cm}^{-2} \AA^{-1}\right)\end{array}$ & $\begin{array}{c}\mathrm{H} \beta \lambda 4861 \\
\left(10^{-13} \mathrm{erg} \mathrm{s}^{-1} \mathrm{~cm}^{-2}\right)\end{array}$ & Observatory \\
\hline 4211.41 & $4.70 \pm 0.31$ & ... & $\mathrm{CrAO}$ \\
\hline 4212.34 & $4.64 \pm 0.15$ & ... & $\mathrm{CrAO}$ \\
\hline 4212.72 & $5.11 \pm 0.20$ & $5.01 \pm 0.11$ & MDM \\
\hline 4212.75 & $4.78 \pm 0.03$ & $\ldots$ & MAGNUM \\
\hline 4213.34 & $5.11 \pm 0.19$ & $\ldots$ & $\mathrm{CrAO}$ \\
\hline 4213.73 & $4.90 \pm 0.20$ & $4.50 \pm 0.09$ & MDM \\
\hline 4214.34 & $4.65 \pm 0.20$ & $\ldots$ & $\mathrm{CrAO}$ \\
\hline 4214.73 & $4.92 \pm 0.20$ & $5.15 \pm 0.11$ & MDM \\
\hline 4215.40 & $4.72 \pm 0.21$ & $\ldots$ & $\mathrm{CrAO}$ \\
\hline 4215.74 & $4.84 \pm 0.20$ & $4.84 \pm 0.10$ & MDM \\
\hline 4216.32 & $5.15 \pm 0.21$ & $\ldots$ & $\mathrm{CrAO}$ \\
\hline 4216.73 & $4.73 \pm 0.19$ & $5.09 \pm 0.11$ & MDM \\
\hline 4217.35 & $4.49 \pm 0.23$ & $\ldots$ & $\mathrm{CrAO}$ \\
\hline 4217.73 & $4.73 \pm 0.19$ & $5.22 \pm 0.11$ & MDM \\
\hline 4218.31 & $4.58 \pm 0.19$ & $\ldots$ & $\mathrm{CrAO}$ \\
\hline 4218.80 & $4.31 \pm 0.19$ & $4.74 \pm 0.10$ & MDM \\
\hline 4218.91 & $4.70 \pm 0.04$ & $\ldots$ & MAGNUM \\
\hline 4219.32 & $4.73 \pm 0.21$ & $\ldots$ & $\mathrm{CrAO}$ \\
\hline 4219.40 & $4.71 \pm 0.16$ & $\ldots$ & UNebr. \\
\hline 4219.83 & $5.06 \pm 0.20$ & $4.70 \pm 0.10$ & MDM \\
\hline 4220.31 & $4.78 \pm 0.25$ & $\ldots$ & $\mathrm{CrAO}$ \\
\hline 4220.40 & $5.05 \pm 0.16$ & $\ldots$ & UNebr. \\
\hline 4220.74 & $5.18 \pm 0.20$ & $4.89 \pm 0.10$ & MDM \\
\hline 4221.35 & $5.23 \pm 0.38$ & $\ldots$ & $\mathrm{CrAO}$ \\
\hline 4221.74 & $4.91 \pm 0.20$ & $4.75 \pm 0.10$ & MDM \\
\hline 4221.99 & $4.78 \pm 0.08$ & $\ldots$ & MAGNUM \\
\hline 4222.40 & $5.08 \pm 0.26$ & $\ldots$ & $\mathrm{CrAO}$ \\
\hline 4222.74 & $4.91 \pm 0.20$ & $4.83 \pm 0.10$ & MDM \\
\hline 4223.38 & $4.47 \pm 0.25$ & $\ldots$ & $\mathrm{CrAO}$ \\
\hline 4223.74 & $4.73 \pm 0.19$ & $5.22 \pm 0.11$ & MDM \\
\hline
\end{tabular}


Table 1-Continued

\begin{tabular}{|c|c|c|c|}
\hline $\begin{array}{c}\text { JD } \\
(-2450000)\end{array}$ & $\begin{array}{c}F_{\lambda}(5100 \AA)^{\mathrm{a}} \\
\left(10^{-15} \mathrm{erg} \mathrm{s}^{-1} \mathrm{~cm}^{-2} \AA^{-1}\right)\end{array}$ & $\begin{array}{c}\mathrm{H} \beta \lambda 4861 \\
\left(10^{-13} \mathrm{erg} \mathrm{s}^{-1} \mathrm{~cm}^{-2}\right)\end{array}$ & Observatory \\
\hline 4224.37 & $5.12 \pm 0.19$ & $\ldots$ & $\mathrm{CrAO}$ \\
\hline 4224.73 & $4.81 \pm 0.20$ & $5.41 \pm 0.11$ & MDM \\
\hline 4225.35 & $4.46 \pm 0.16$ & $\ldots$ & $\mathrm{CrAO}$ \\
\hline 4225.80 & $4.30 \pm 0.19$ & $4.56 \pm 0.10$ & MDM \\
\hline 4225.88 & $4.43 \pm 0.03$ & $\ldots$ & MAGNUM \\
\hline 4226.28 & $4.46 \pm 0.20$ & $\ldots$ & $\mathrm{CrAO}$ \\
\hline 4226.75 & $3.88 \pm 0.18$ & $4.55 \pm 0.10$ & MDM \\
\hline 4227.43 & $4.28 \pm 0.23$ & $\ldots$ & $\mathrm{CrAO}$ \\
\hline 4227.74 & $4.12 \pm 0.19$ & $4.58 \pm 0.10$ & MDM \\
\hline 4228.80 & $3.81 \pm 0.18$ & $4.54 \pm 0.10$ & MDM \\
\hline 4229.37 & $3.91 \pm 0.15$ & $\ldots$ & $\mathrm{CrAO}$ \\
\hline 4229.77 & $3.91 \pm 0.18$ & $4.31 \pm 0.09$ & MDM \\
\hline 4230.73 & $4.38 \pm 0.19$ & $4.36 \pm 0.09$ & MDM \\
\hline 4231.36 & $4.07 \pm 0.16$ & $\ldots$ & $\mathrm{CrAO}$ \\
\hline 4231.59 & $4.58 \pm 0.28$ & $\ldots$ & UNebr. \\
\hline 4231.75 & $4.81 \pm 0.20$ & $4.61 \pm 0.10$ & MDM \\
\hline 4232.28 & $4.47 \pm 0.16$ & $\ldots$ & $\mathrm{CrAO}$ \\
\hline 4232.38 & $4.22 \pm 0.19$ & 4.53ata & UNebr. \\
\hline 4232.73 & $4.51 \pm 0.15$ & $0.00 \pm 0.09$ & MDM \\
\hline 4233.32 & $4.88 \pm 0.18$ & $\ldots$ & $\mathrm{CrAO}$ \\
\hline 4233.44 & $4.64 \pm 0.19$ & 4.72ata & UNebr. \\
\hline 4233.73 & $4.17 \pm 0.16$ & $0.00 \pm 0.10$ & MDM \\
\hline 4234.32 & $4.51 \pm 0.19$ & 4.69ata & $\mathrm{CrAO}$ \\
\hline 4234.73 & $4.29 \pm 0.03$ & $0.00 \pm 0.10$ & MDM \\
\hline 4234.85 & $4.35 \pm 0.14$ & $\ldots$ & MAGNUM \\
\hline 4235.31 & $4.80 \pm 0.36$ & $\ldots$ & $\mathrm{CrAO}$ \\
\hline 4235.46 & $4.43 \pm 0.19$ & 4.56ata & UNebr. \\
\hline 4235.73 & $4.53 \pm 0.15$ & $0.00 \pm 0.10$ & MDM \\
\hline 4236.31 & $3.96 \pm 0.18$ & 4.54ata & $\mathrm{CrAO}$ \\
\hline 4236.73 & $4.17 \pm 0.14$ & $0.00 \pm 0.09$ & MDM \\
\hline
\end{tabular}


Table 1-Continued

\begin{tabular}{|c|c|c|c|}
\hline $\begin{array}{c}\text { JD } \\
(-2450000)\end{array}$ & $\begin{array}{c}F_{\lambda}(5100 \AA)^{\mathrm{a}} \\
\left(10^{-15} \mathrm{erg} \mathrm{s}^{-1} \mathrm{~cm}^{-2} \AA^{-1}\right)\end{array}$ & $\begin{array}{c}\mathrm{H} \beta \lambda 4861 \\
\left(10^{-13} \mathrm{erg} \mathrm{s}^{-1} \mathrm{~cm}^{-2}\right)\end{array}$ & Observatory \\
\hline 4237.31 & $4.13 \pm 0.28$ & $\ldots$ & $\mathrm{CrAO}$ \\
\hline 4237.52 & $3.94 \pm 0.18$ & 4.28ata & UNebr. \\
\hline 4237.73 & $4.38 \pm 0.17$ & $0.00 \pm 0.09$ & MDM \\
\hline 4238.49 & $4.16 \pm 0.19$ & 4.42ata & UNebr. \\
\hline 4238.73 & $4.05 \pm 0.06$ & $0.00 \pm 0.09$ & MDM \\
\hline 4238.93 & $4.19 \pm 0.17$ & $\ldots$ & MAGNUM \\
\hline 4239.36 & $4.59 \pm 0.22$ & $\ldots$ & $\mathrm{CrAO}$ \\
\hline 4239.43 & $4.34 \pm 0.18$ & $\ldots$ & UNebr. \\
\hline 4239.75 & $3.81 \pm 0.18$ & $4.40 \pm 0.09$ & MDM \\
\hline 4240.29 & $3.69 \pm 0.14$ & $\ldots$ & $\mathrm{CrAO}$ \\
\hline 4240.47 & $3.69 \pm 0.18$ & $\ldots$ & UNebr. \\
\hline 4240.72 & $3.97 \pm 0.18$ & $4.37 \pm 0.09$ & MDM \\
\hline 4241.31 & $3.74 \pm 0.16$ & $\ldots$ & $\mathrm{CrAO}$ \\
\hline 4241.38 & $3.92 \pm 0.24$ & $\ldots$ & UNebr. \\
\hline 4241.73 & $3.75 \pm 0.18$ & $4.30 \pm 0.09$ & MDM \\
\hline 4242.29 & $3.75 \pm 0.14$ & $\ldots$ & $\mathrm{CrAO}$ \\
\hline 4242.74 & $3.56 \pm 0.18$ & $3.91 \pm 0.08$ & MDM \\
\hline 4243.29 & $3.35 \pm 0.19$ & $\ldots$ & $\mathrm{CrAO}$ \\
\hline 4243.74 & $3.39 \pm 0.18$ & $4.05 \pm 0.09$ & MDM \\
\hline 4244.78 & $3.68 \pm 0.18$ & $3.90 \pm 0.08$ & MDM \\
\hline 4245.36 & $4.38 \pm 0.25$ & $\ldots$ & $\mathrm{CrAO}$ \\
\hline 4245.75 & $4.36 \pm 0.19$ & $4.03 \pm 0.09$ & MDM \\
\hline 4245.77 & $4.21 \pm 0.06$ & $\ldots$ & MAGNUM \\
\hline 4246.34 & $4.95 \pm 0.18$ & $\ldots$ & $\mathrm{CrAO}$ \\
\hline 4246.40 & $4.49 \pm 0.17$ & $\ldots$ & UNebr. \\
\hline 4246.73 & $4.06 \pm 0.19$ & $4.12 \pm 0.09$ & MDM \\
\hline 4247.74 & $4.57 \pm 0.19$ & $4.20 \pm 0.09$ & MDM \\
\hline 4248.33 & $4.50 \pm 0.21$ & $\ldots$ & $\mathrm{CrAO}$ \\
\hline 4248.73 & $4.50 \pm 0.19$ & $4.48 \pm 0.09$ & MDM \\
\hline 4249.45 & $4.36 \pm 0.22$ & $\ldots$ & $\mathrm{CrAO}$ \\
\hline
\end{tabular}


Table 1-Continued

\begin{tabular}{|c|c|c|c|}
\hline $\begin{array}{c}\text { JD } \\
(-2450000)\end{array}$ & $\begin{array}{c}F_{\lambda}(5100 \AA)^{\mathrm{a}} \\
\left(10^{-15} \mathrm{erg} \mathrm{s}^{-1} \mathrm{~cm}^{-2} \AA^{-1}\right)\end{array}$ & $\begin{array}{c}\mathrm{H} \beta \lambda 4861 \\
\left(10^{-13} \mathrm{erg} \mathrm{s}^{-1} \mathrm{~cm}^{-2}\right)\end{array}$ & Observatory \\
\hline 4249.73 & $4.40 \pm 0.19$ & $4.52 \pm 0.09$ & MDM \\
\hline 4250.41 & $3.56 \pm 0.33$ & $\ldots$ & $\mathrm{CrAO}$ \\
\hline 4250.74 & $4.07 \pm 0.19$ & $4.44 \pm 0.09$ & MDM \\
\hline 4251.32 & $3.74 \pm 0.30$ & $\ldots$ & $\mathrm{CrAO}$ \\
\hline 4251.74 & $4.02 \pm 0.19$ & $4.39 \pm 0.09$ & MDM \\
\hline 4252.39 & $4.47 \pm 0.27$ & $\ldots$ & $\mathrm{CrAO}$ \\
\hline 4252.73 & $4.15 \pm 0.19$ & $4.22 \pm 0.09$ & MDM \\
\hline 4252.88 & $4.17 \pm 0.05$ & $\ldots$ & MAGNUM \\
\hline 4253.73 & $4.44 \pm 0.19$ & $4.19 \pm 0.09$ & MDM \\
\hline 4254.35 & $4.62 \pm 0.16$ & $\ldots$ & $\mathrm{CrAO}$ \\
\hline 4254.39 & $4.52 \pm 0.17$ & $\ldots$ & UNebr. \\
\hline 4255.38 & $4.73 \pm 0.24$ & ... & $\mathrm{CrAO}$ \\
\hline 4255.76 & $4.40 \pm 0.19$ & $4.50 \pm 0.09$ & MDM \\
\hline 4256.35 & $5.02 \pm 0.14$ & $\ldots$ & $\mathrm{CrAO}$ \\
\hline 4256.71 & $4.75 \pm 0.19$ & $4.56 \pm 0.10$ & MDM \\
\hline 4257.40 & $4.80 \pm 0.17$ & $\ldots$ & $\mathrm{CrAO}$ \\
\hline 4257.74 & $4.64 \pm 0.19$ & $4.47 \pm 0.09$ & MDM \\
\hline 4258.35 & $4.52 \pm 0.16$ & $\ldots$ & $\mathrm{CrAO}$ \\
\hline 4258.50 & $5.06 \pm 0.32$ & $\ldots$ & UNebr. \\
\hline 4258.76 & $4.83 \pm 0.20$ & $4.91 \pm 0.10$ & MDM \\
\hline 4259.34 & $5.15 \pm 0.17$ & $\ldots$ & $\mathrm{CrAO}$ \\
\hline 4259.75 & $4.92 \pm 0.20$ & $4.97 \pm 0.10$ & MDM \\
\hline 4259.84 & $4.81 \pm 0.13$ & $\ldots$ & MAGNUM \\
\hline 4260.30 & $4.96 \pm 0.17$ & $\ldots$ & $\mathrm{CrAO}$ \\
\hline 4260.75 & $4.36 \pm 0.19$ & $4.70 \pm 0.10$ & MDM \\
\hline 4261.31 & $5.08 \pm 0.16$ & $\ldots$ & $\mathrm{CrAO}$ \\
\hline 4261.42 & $5.15 \pm 0.14$ & $\ldots$ & UNebr. \\
\hline 4261.74 & $4.82 \pm 0.20$ & $5.16 \pm 0.11$ & MDM \\
\hline 4262.30 & $4.78 \pm 0.16$ & $\ldots$ & $\mathrm{CrAO}$ \\
\hline 4262.74 & $4.39 \pm 0.19$ & $4.72 \pm 0.10$ & MDM \\
\hline
\end{tabular}


Table 1-Continued

\begin{tabular}{|c|c|c|c|}
\hline $\begin{array}{c}\mathrm{JD} \\
(-2450000)\end{array}$ & $\begin{array}{c}F_{\lambda}(5100 \AA)^{\mathrm{a}} \\
\left(10^{-15} \mathrm{erg} \mathrm{s}^{-1} \mathrm{~cm}^{-2} \AA^{-1}\right)\end{array}$ & $\begin{array}{c}\mathrm{H} \beta \lambda 4861 \\
\left(10^{-13} \mathrm{erg} \mathrm{s}^{-1} \mathrm{~cm}^{-2}\right)\end{array}$ & Observatory \\
\hline 4263.35 & $4.68 \pm 0.17$ & $\ldots$ & $\mathrm{CrAO}$ \\
\hline 4263.39 & $4.47 \pm 0.22$ & $\ldots$ & UNebr. \\
\hline 4263.72 & $4.52 \pm 0.19$ & $5.01 \pm 0.11$ & MDM \\
\hline 4263.88 & $4.61 \pm 0.11$ & $\ldots$ & MAGNUM \\
\hline 4264.76 & $4.73 \pm 0.19$ & $5.12 \pm 0.11$ & MDM \\
\hline 4265.76 & $5.43 \pm 0.20$ & $5.15 \pm 0.11$ & MDM \\
\hline 4266.34 & $4.84 \pm 0.27$ & $5.18 \pm 0.17$ & $\mathrm{CrAO}$ \\
\hline 4266.76 & $5.55 \pm 0.21$ & $5.29 \pm 0.11$ & MDM \\
\hline 4267.30 & $4.43 \pm 0.26$ & $5.43 \pm 0.18$ & $\mathrm{CrAO}$ \\
\hline 4267.75 & $4.55 \pm 0.19$ & $5.24 \pm 0.11$ & MDM \\
\hline 4268.29 & $4.33 \pm 0.26$ & $5.34 \pm 0.18$ & $\mathrm{CrAO}$ \\
\hline 4268.75 & $4.38 \pm 0.19$ & $5.17 \pm 0.11$ & MDM \\
\hline 4269.32 & $4.57 \pm 0.26$ & $5.16 \pm 0.17$ & $\mathrm{CrAO}$ \\
\hline 4269.75 & $4.56 \pm 0.19$ & $5.39 \pm 0.11$ & MDM \\
\hline 4269.84 & $4.37 \pm 0.06$ & $\ldots$ & MAGNUM \\
\hline 4270.36 & $5.09 \pm 0.27$ & $5.12 \pm 0.17$ & $\mathrm{CrAO}$ \\
\hline 4270.37 & $4.37 \pm 0.20$ & $\ldots$ & UNebr. \\
\hline 4271.31 & $4.52 \pm 0.26$ & $5.38 \pm 0.18$ & $\mathrm{CrAO}$ \\
\hline 4272.85 & $4.54 \pm 0.04$ & $\ldots$ & MAGNUM \\
\hline 4274.31 & $4.78 \pm 0.26$ & $5.16 \pm 0.17$ & $\mathrm{CrAO}$ \\
\hline 4275.81 & $4.31 \pm 0.03$ & $\ldots$ & MAGNUM \\
\hline 4276.41 & $4.80 \pm 0.20$ & $\ldots$ & UNebr. \\
\hline 4277.29 & $4.42 \pm 0.26$ & $4.79 \pm 0.16$ & $\mathrm{CrAO}$ \\
\hline 4278.29 & $4.01 \pm 0.25$ & $4.99 \pm 0.16$ & $\mathrm{CrAO}$ \\
\hline 4278.48 & $3.66 \pm 0.37$ & $\ldots$ & UNebr. \\
\hline 4278.84 & $4.14 \pm 0.06$ & $\ldots$ & MAGNUM \\
\hline 4279.31 & $4.33 \pm 0.26$ & $4.74 \pm 0.16$ & $\mathrm{CrAO}$ \\
\hline 4279.32 & $4.22 \pm 0.21$ & $\ldots$ & $\mathrm{CrAO}$ \\
\hline 4280.32 & $4.61 \pm 0.26$ & $4.87 \pm 0.16$ & $\mathrm{CrAO}$ \\
\hline 4280.34 & $4.38 \pm 0.43$ & $\ldots$ & $\mathrm{CrAO}$ \\
\hline
\end{tabular}


Table 1-Continued

\begin{tabular}{|c|c|c|c|}
\hline $\begin{array}{c}\text { JD } \\
(-2450000)\end{array}$ & $\begin{array}{c}F_{\lambda}(5100 \AA)^{\mathrm{a}} \\
\left(10^{-15} \mathrm{erg} \mathrm{s}^{-1} \mathrm{~cm}^{-2} \AA^{-1}\right)\end{array}$ & $\begin{array}{c}\mathrm{H} \beta \lambda 4861 \\
\left(10^{-13} \mathrm{erg} \mathrm{s}^{-1} \mathrm{~cm}^{-2}\right)\end{array}$ & Observatory \\
\hline 4281.32 & $4.34 \pm 0.24$ & $0.00 \pm 0.16$ & $\mathrm{CrAO}$ \\
\hline 4281.32 & $4.38 \pm 0.26$ & 4.88ata & $\mathrm{CrAO}$ \\
\hline 4282.33 & $3.96 \pm 0.24$ & $\ldots$ & $\mathrm{CrAO}$ \\
\hline 4282.35 & $4.22 \pm 0.25$ & $4.47 \pm 0.15$ & $\mathrm{CrAO}$ \\
\hline 4283.31 & $3.78 \pm 0.25$ & $4.63 \pm 0.15$ & $\mathrm{CrAO}$ \\
\hline 4283.33 & $3.75 \pm 0.24$ & $\cdots$ & $\mathrm{CrAO}$ \\
\hline 4283.42 & $3.56 \pm 0.20$ & $\cdots$ & UNebr. \\
\hline 4284.30 & $3.64 \pm 0.24$ & $4.50 \pm 0.15$ & $\mathrm{CrAO}$ \\
\hline 4284.31 & $3.87 \pm 0.18$ & $\cdots$ & $\mathrm{CrAO}$ \\
\hline 4285.77 & $3.70 \pm 0.09$ & $\cdots$ & MAGNUM \\
\hline 4289.29 & $4.20 \pm 0.25$ & $4.22 \pm 0.14$ & $\mathrm{CrAO}$ \\
\hline 4289.45 & $3.65 \pm 0.25$ & $\cdots$ & UNebr. \\
\hline 4290.30 & $3.85 \pm 0.25$ & $4.41 \pm 0.15$ & $\mathrm{CrAO}$ \\
\hline 4290.40 & $4.27 \pm 0.22$ & $\ldots$ & UNebr. \\
\hline 4291.30 & $3.53 \pm 0.24$ & $4.34 \pm 0.14$ & $\mathrm{CrAO}$ \\
\hline 4294.29 & $4.63 \pm 0.18$ & $\ldots$ & $\mathrm{CrAO}$ \\
\hline 4295.32 & $4.51 \pm 0.34$ & $\cdots$ & $\mathrm{CrAO}$ \\
\hline 4296.29 & $4.35 \pm 0.26$ & $4.67 \pm 0.15$ & $\mathrm{CrAO}$ \\
\hline 4296.30 & $4.57 \pm 0.17$ & $\cdots$ & $\mathrm{CrAO}$ \\
\hline 4298.32 & $4.78 \pm 0.26$ & $4.38 \pm 0.14$ & $\mathrm{CrAO}$ \\
\hline 4299.28 & $4.29 \pm 0.26$ & $4.65 \pm 0.15$ & $\mathrm{CrAO}$ \\
\hline 4299.31 & $4.87 \pm 0.16$ & $\cdots$ & $\mathrm{CrAO}$ \\
\hline 4300.28 & $4.14 \pm 0.25$ & $4.68 \pm 0.15$ & $\mathrm{CrAO}$ \\
\hline 4304.79 & $4.66 \pm 0.11$ & $\cdots$ & MAGNUM \\
\hline 4311.76 & $4.79 \pm 0.09$ & . & MAGNUM \\
\hline
\end{tabular}

aThis column contains the average continuum flux density measured at restframe $\sim 5100 \AA$ from spectroscopic observations as well as the $V$-band flux from photometric observations. Spectroscopic and photometric fluxes were intercalibrated and merged to create a single continuum light curve (see Section 2.3). 
Table 2. Light Curve Statistics

\begin{tabular}{|c|c|c|c|c|c|c|c|}
\hline \multirow{2}{*}{$\begin{array}{c}\text { Time } \\
\text { Series } \\
(1)\end{array}$} & \multirow[b]{2}{*}{$\begin{array}{l}N \\
(2)\end{array}$} & \multicolumn{2}{|c|}{$\begin{array}{c}\text { Sampling } \\
\text { Interval(days) }\end{array}$} & \multirow{2}{*}{$\begin{array}{l}\text { Mean } \\
\text { Flux }^{\mathrm{a}} \\
(5)\end{array}$} & \multirow{2}{*}{$\begin{array}{c}\text { Mean } \\
\text { Fractional } \\
\text { Error } \\
(6)\end{array}$} & \multirow[b]{2}{*}{$\begin{array}{c}F_{\mathrm{var}} \\
(7)\end{array}$} & \multirow[b]{2}{*}{$\begin{array}{c}R_{\max } \\
(8)\end{array}$} \\
\hline & & $\begin{array}{l}\langle T\rangle \\
(3)\end{array}$ & $\begin{array}{c}T_{\text {median }} \\
\quad(4)\end{array}$ & & & & \\
\hline $5100 \AA$ & 186 & 0.71 & 0.56 & $4.5 \pm 0.4$ & 0.04 & 0.09 & $1.69 \pm 0.11$ \\
\hline $\mathrm{H} \beta$ & 100 & 1.17 & 1.00 & $4.7 \pm 0.3$ & 0.02 & 0.07 & $1.39 \pm 0.04$ \\
\hline
\end{tabular}

${ }^{a}$ Same flux units as Table 1 for $5100 \AA$ continuum and $\mathrm{H} \beta$, respectively. 
Table 3. Reverberation Results

\begin{tabular}{cc}
\hline \hline Parameter & Value \\
$(1)$ & $(2)$ \\
\hline$\tau_{\text {cent }}$ & $1.87_{-0.50}^{+0.54}$ days \\
$\tau_{\text {peak }}$ & $2.60_{-1.40}^{+0.79}$ days \\
$\sigma_{\text {line }}($ mean $)$ & $1045 \pm 4 \mathrm{~km} \mathrm{~s}^{-1}$ \\
FWHM (mean) & $799 \pm 2 \mathrm{~km} \mathrm{~s}^{-1}$ \\
$\sigma_{\text {line }}(\mathrm{rms})$ & $927 \pm 64 \mathrm{~km} \mathrm{~s}^{-1}$ \\
$\mathrm{FWHM} \mathrm{(rms)}$ & $1034 \pm 41 \mathrm{~km} \mathrm{~s}^{-1}$ \\
$M_{\mathrm{BH}}{ }^{\mathrm{a}}$ & $\left(1.73_{-0.52}^{+0.55}\right) \times 10^{6} M_{\odot}$ \\
$M_{\mathrm{BH}-\mathrm{rad}}$ & $\left(1.24_{-0.56}^{+0.57}\right) \times 10^{6} M_{\odot}$ \\
\hline
\end{tabular}

a Using Onken et al. (2004) calibration.

${ }^{\mathrm{b}}$ Using Marconi et al. (2008) calibration. 


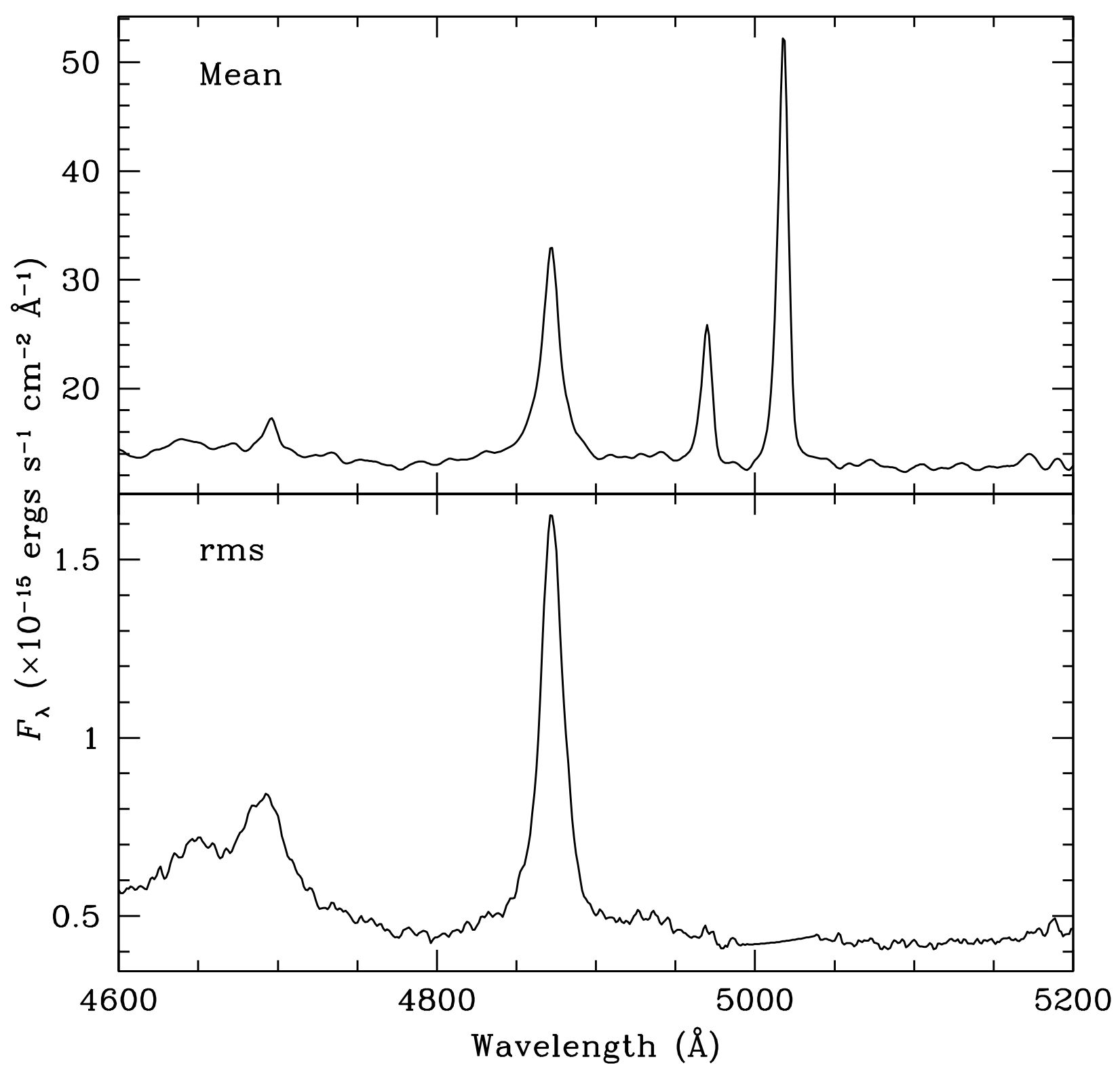

Fig. 1.- Mean and rms spectra of NGC 4051 from MDM observations. The rms spectrum was formed after removing the [O III] $\lambda 4959$ and $[\mathrm{O}$ III] $\lambda 5007$ narrow emission lines. The variability signature of $\mathrm{H} \beta$ is clearly visible in the rms spectrum, and the large increase in rms flux shortward of $4800 \AA$ is due to variations in the broad He II $\lambda 4686$ emission line. 


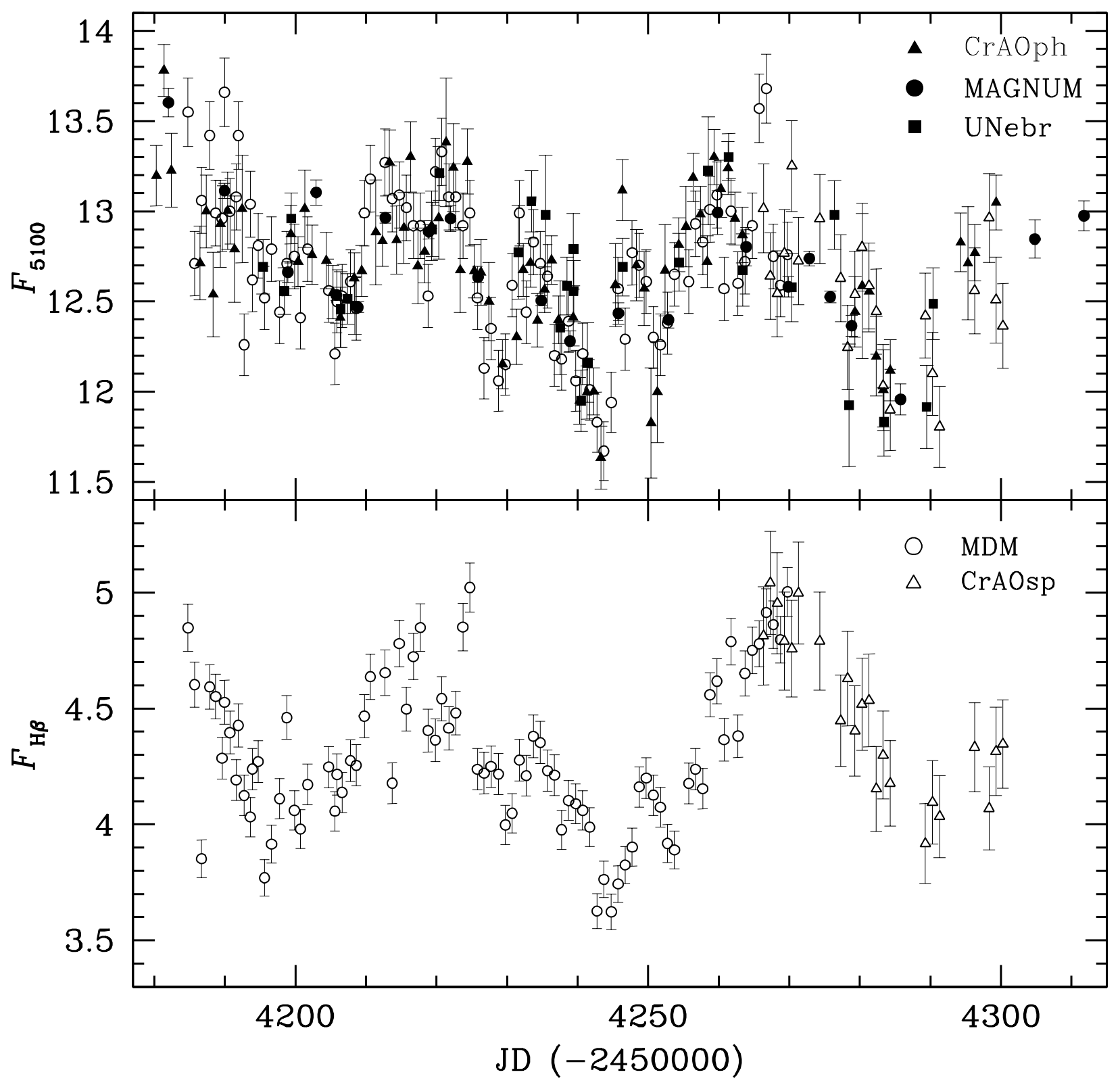

Fig. 2. - Light curves showing complete set of observations from all four sources. The top panel shows the $5100 \AA$ continuum flux in units of $10^{-15} \mathrm{erg} \mathrm{s}^{-1} \mathrm{~cm}^{-2} \AA^{-1}$, while the bottom is the $\mathrm{H} \beta \lambda 4861$ line flux in units of $10^{-13} \mathrm{erg} \mathrm{s}^{-1} \mathrm{~cm}^{-2}$. The open triangles (CrAOsp) correspond to spectroscopic observations taken at $\mathrm{CrAO}$, while the closed triangles (CrAOph) represent photometric observations from $\mathrm{CrAO}$. 


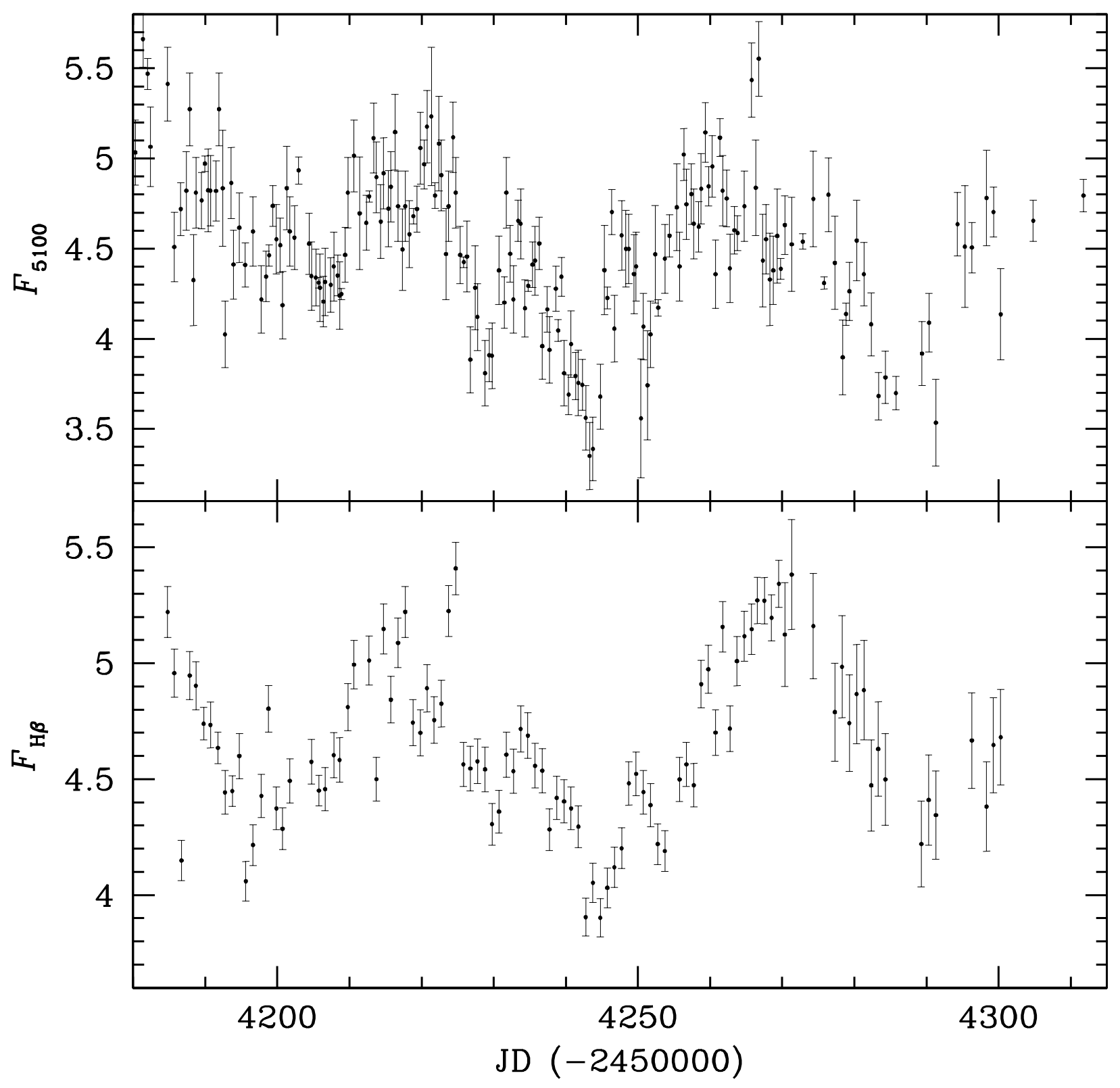

Fig. 3.- Same as Fig. 2 except data from all sources have been merged and closely spaced observations binned such that weighted averages were calculated for continuum observations separated by less than 0.25 day and $\mathrm{H} \beta$ observations separated by less than 0.5 day. 


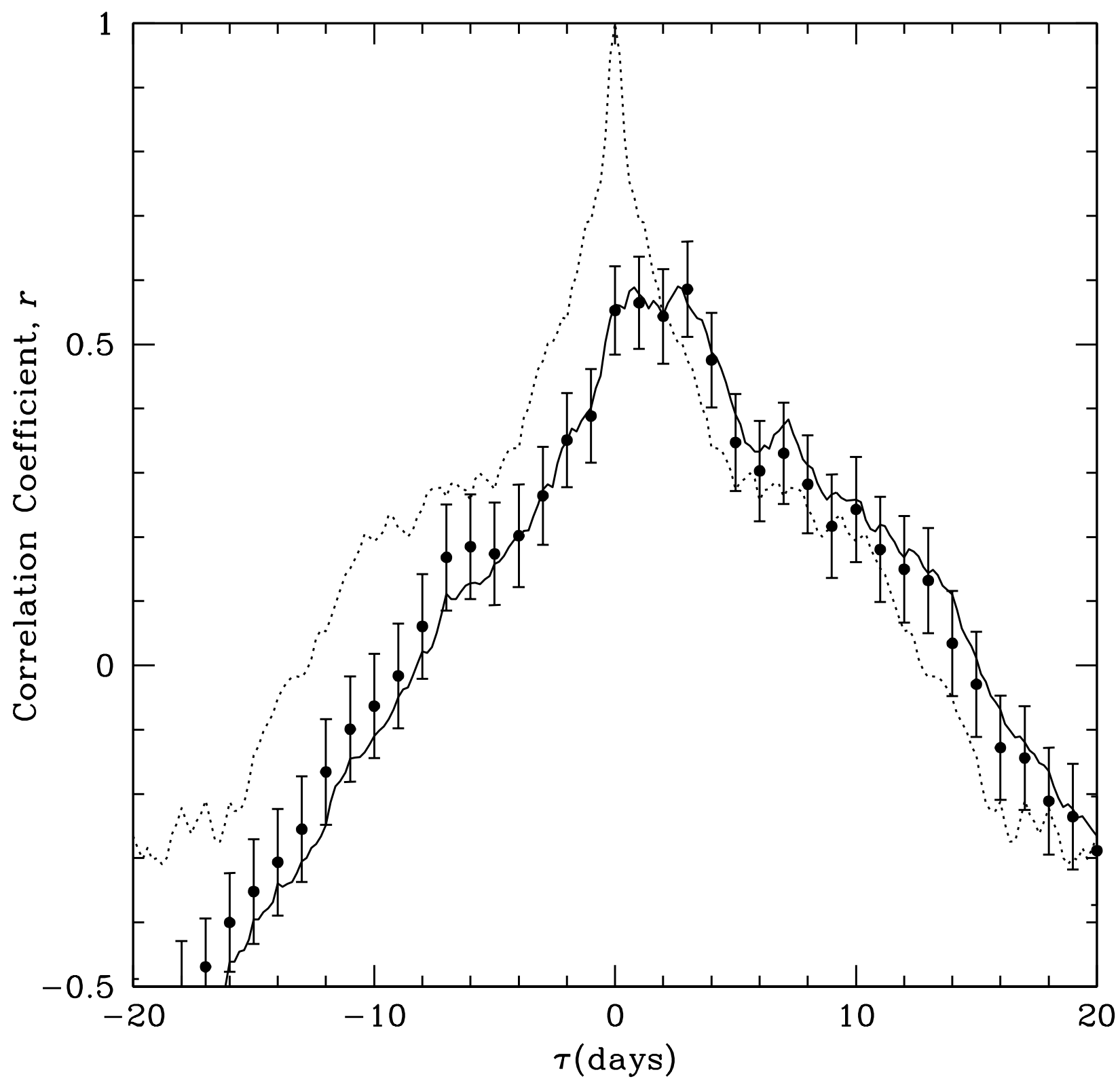

Fig. 4.- Cross correlation function (CCF; solid line), discrete correlation function (DCF; filled circles), and auto correlation function (ACF; dotted line) from time series analysis of the continuum and $\mathrm{H} \beta$ light curves of NGC 4051 shown in Fig. 3 , 


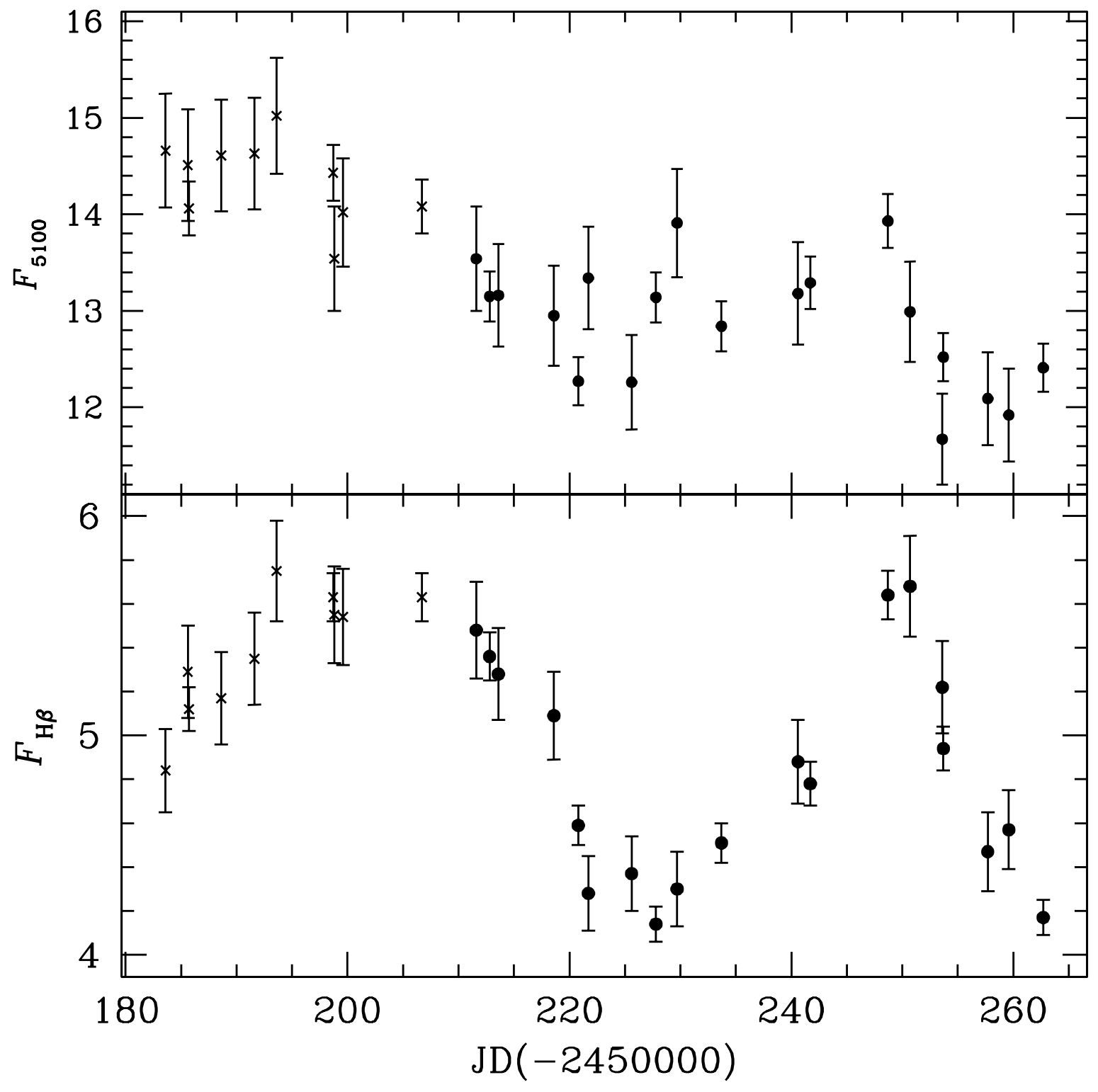

Fig. 5.- Optical continuum and $\mathrm{H} \beta$ light curves reproduced from P00. Points with X's represent those that were excluded from the new time series analysis of this light curve described in Section 3, Units are the same as in Fig. 2, 


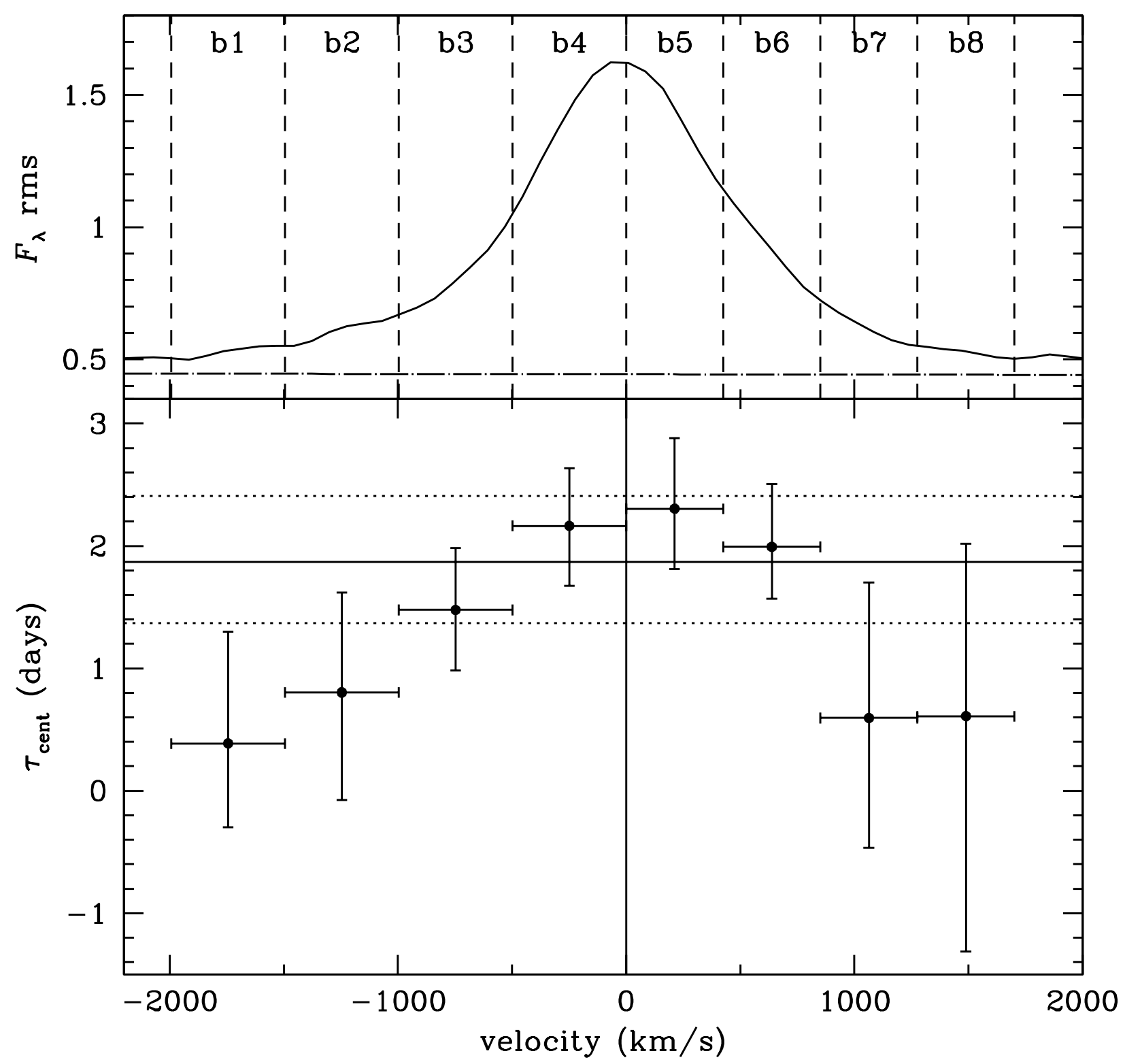

Fig. 6.- Velocity-resolved $\mathrm{H} \beta$ rms spectrum profile (top) and time-delay measurements (bottom) for NGC 4051. Vertical dashed lines plotted on the line profile (top) and error bars on the lag measurements in the velocity direction (bottom) show the bin size, with each bin labeled by number in the top panel. Error bars on the lag measurements are determined similarly to those for the mean BLR lag. The horizontal solid and dotted lines in the bottom panel show the mean BLR centroid lag and associated errors, calculated in Section 2.4, while the horizontal dotted-dashed line in the top panel represents the linearly-fit continuum level. Flux units are the same as in Fig. 1. 


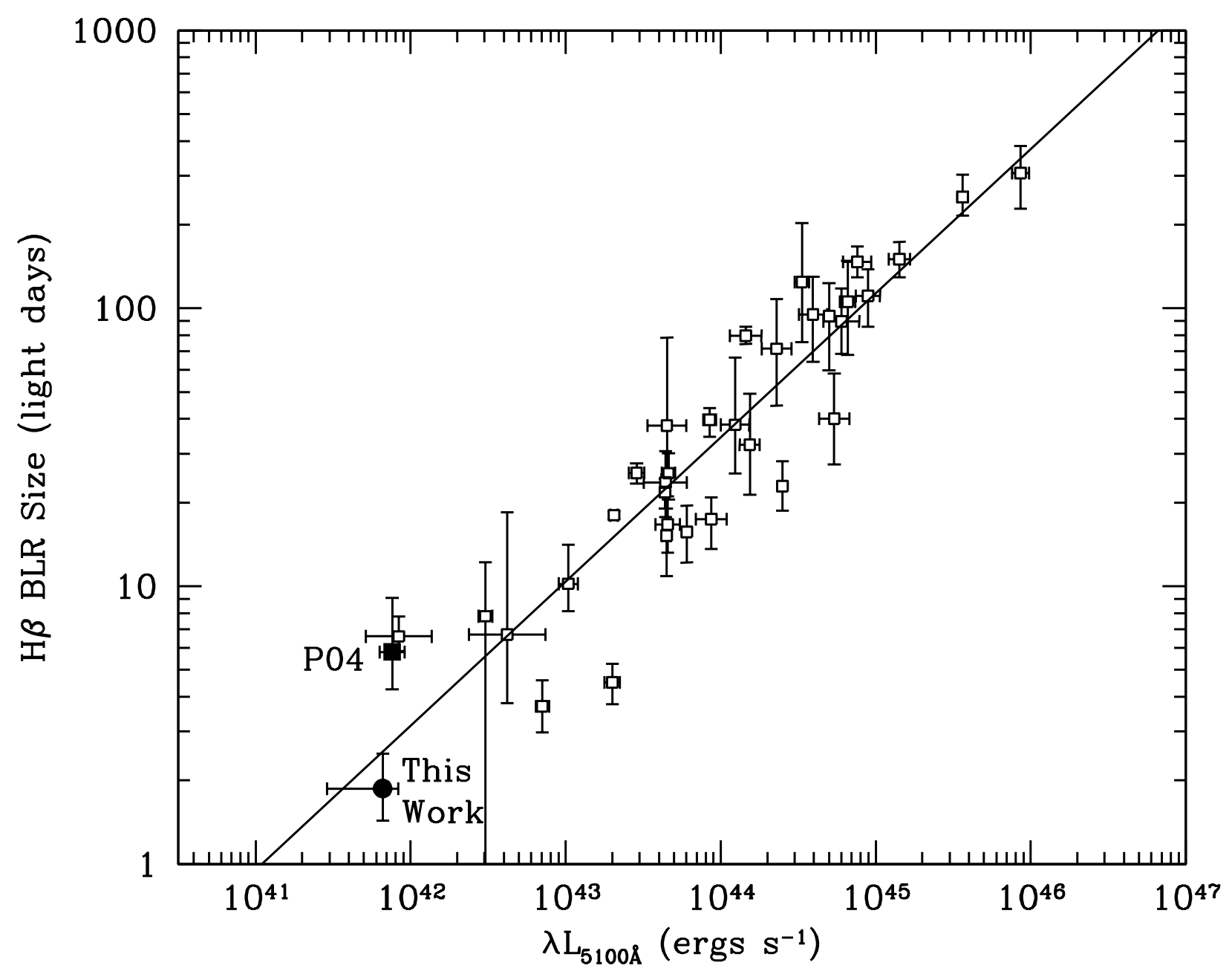

Fig. 7.- Most recently calibrated $R_{\mathrm{BLR}}-L$ relation (Bentz et al. 2009a, solid line). The filled square shows the location of NGC 4051 based on results from Peterson et al. (2004) and used by Bentz et al. The filled circle shows the new lag measurement of 1.87 days presented in this work at the luminosity calculated using the Tully-Fisher distance to NGC 4051 of Russell (2003): the error bar in luminosity reflects both the range of flux variations, as for all of the other data points, plus the uncertainty due to the distance, added in quadrature. The error bar is asymmetric, as we favor the larger distance. Open squares represent other objects from Bentz et al. (2009a). 